\title{
Boosting perturbative QCD stability in quarkonium production
}

\section{Hua-Sheng Shao}

Laboratoire de Physique Théorique et Hautes Energies (LPTHE), UMR 7589, Sorbonne Université et CNRS, 4 place Jussieu, 75252 Paris Cedex 05, France

E-mail: huasheng.shao@lpthe.jussieu.fr

ABSTRACT: The aim of this paper is to introduce a general way to stabilize the perturbative QCD computations of heavy quarkonium production in the boosted or high-momentum transferring region with tree-level generators only. Such an approach is possible by properly taking into account the power-enhanced perturbative contributions in a soft and collinear safe manner without requiring any complete higher-order computations. The complicated NLO results for inclusive quarkonium hadroproduction can be well reproduced within our approach based on a tree-level generator HELAC-OnIA. We have applied it to estimate the last missing leading-twist contribution from the spin-triplet color-singlet $\mathrm{S}$-wave production at $\mathcal{O}\left(\alpha_{s}^{5}\right)$, which is a NNLO term in the $\alpha_{s}$ expansion for the quarkonium $P_{T}$ spectrum. We conclude that the missing NNLO contribution will not change the order of the magnitude of the short-distance coefficient. Such an approach is also quite appealing as it foresees broad applications in quarkonium-associated production processes, which are mostly absent of complete higher-order computations and fragmentation functions.

KeYwords: NLO Computations, QCD Phenomenology

ARXiv EPrint: 1809.02369 


\section{Contents}

1 Introduction and motivations 1

2 Remainders of P-wave counterterms 4

3 A first step towards NLO 5

4 A general infrared-safe method $\quad 8$

4.1 Infrared-safe cutoffs 8

4.2 Reproducing NLO results 11

4.2.1 Reproducing ${ }^{3} S_{1}^{[8]} \quad 11$

$\begin{array}{ll}\text { 4.2.2 Reproducing other Fock states } & 11\end{array}$

4.3 Going beyond NLO 13

$\begin{array}{lll}4.4 & \text { Reassessing the charm fragmentation } & 16\end{array}$

$\begin{array}{llr}5 & \text { Summary and outlooks } & 20\end{array}$

A Calculations with HELAC-ONIA 20

$\begin{array}{ll}\text { A.1 Born and counterterms } & 22\end{array}$

A.2 Real terms 23

B Supplemental plots $\quad 24$

\section{Introduction and motivations}

As a class of the simplest hadrons, heavy quarkonium is usually viewed as the "hydrogen atom" in the strong-interaction theory QCD. While the knowledge of the nonperturbative aspect in QCD is still quite limited, heavy quarkonium provides a unique opportunity to probe the quark confinement in QCD by exploring the physics at the scale around the nonperturbative and perturbative boundary. The intrinsic scales of the heavy quark mass $m_{Q}$ and their binding energy $m_{Q} v^{2}$ lie in the perturbative and nonperturbative regimes respectively, where $v$ is the relative velocity between the heavy quark pair in the rest frame of the quarkonium. Due to the smallness of the relative velocity $v$ (e.g. $v^{2} \simeq 0.3$ and $v^{2} \simeq 0.1$ for the charmonium and bottomonium), the relativistic QCD can be reorganized via the operator product expansion in the power counting of $v$. The effective theory was dubbed as non-relativistic QCD (NRQCD) [1]. The reformulation of QCD provides a factorization conjecture for calculating the rates of the quarkonium production and decay. In the case of 


\begin{tabular}{|c|c|c|c|c|}
\hline Power counting & $\eta_{Q}$ & $\psi, \Upsilon$ & $h_{Q}$ & $\chi Q J$ \\
\hline$v^{3}$ & ${ }^{1} S_{0}^{[1]}$ & ${ }^{3} S_{1}^{[1]}$ & - & - \\
$v^{5}$ & - & - & ${ }^{1} P_{1}^{[1]}, S_{0}^{[8]}$ & ${ }^{3} P_{J}^{[1]},{ }^{3} S_{1}^{[8]}$ \\
$v^{7}$ & ${ }^{1} S_{0}^{[8]},{ }^{3} S_{1}^{[8]},{ }^{1} P_{1}^{[8]}$ & ${ }^{1} S_{0}^{[8]},{ }^{3} S_{1}^{[8]},{ }^{3} P_{J}^{[8]}$ & - & - \\
\hline
\end{tabular}

Table 1. The leading power counting of various Fock states contributing to various quarkonium within NRQCD velocity scaling rule [1].

the quarkonium $\mathcal{H}$ production, the (differential) cross section at leading-order (LO) in the QCD strong coupling constant $\alpha_{s}$ can be schematically written as

$$
d \sigma(\mathcal{H})=\sum_{n} d \hat{\sigma}(n)\left\langle\mathcal{O}^{\mathcal{H}}(n)\right\rangle,
$$

where $n$ represents a Fock state, $d \hat{\sigma}(n)$ is a perturbatively calculable short-distance coefficient (SDC) with the heavy quark pair in the Fock state $n$ and $\left\langle\mathcal{O}^{\mathcal{H}}(n)\right\rangle$ is the vacuum expectation number of an operator $\mathcal{O}^{\mathcal{H}}(n)$. If the factorization formula eq. (1.1) holds, the nonperturbative long-distance matrix element (LDME) $\left\langle\mathcal{O}^{\mathcal{H}}(n)\right\rangle$ is independent of quarkonium production process as well as the production environment. The universal LDMEs, which are analogous to the parton-distribution functions (PDFs) in the perturbative QCD factorization, are to be determined from a subset of the experimental data and to predict all of the rest experimental measurements. They have the probability explanations at LO, while LDMEs depend on the renormalization scheme and they are not physical objects.

The prediction power of eq. (1.1) heavily relies on the perturbative convergences of $v^{2}$ and $\alpha_{s}$ in $d \sigma(\mathcal{H})$. The leading power counting of various Fock states up to $\mathcal{O}\left(v^{7}\right)$ for S-wave and $\mathrm{P}$-wave quarkonia is listed in table 1 according to the NRQCD velocity scaling rule [1]. The convergence in $v^{2}$ can be improved by including the relativistic corrections. However, the prices to pay are that one has to introduce more nonperturbative LDMEs that can not be determined from the first principle, and the good relations like heavy-quark spin symmetry holding at LO in $v$ will be violated too.

The most subtle part is the $\alpha_{s}$ stability in the SDCs $d \hat{\sigma}(n)$, which is the main point to be discussed in this paper. For the high-transverse-momentum $\left(P_{T}\right)$ quarkonium production at a high-energy hadron collider, it was found that ${ }^{3} S_{1}^{[1]}$ receives a giant $\mathrm{K}$ factor from QCD corrections to its SDC a decade ago [2], which was understood by the fact that due to the quantum number conservation, there is a factor $\alpha_{s} \frac{P_{T}^{2}}{4 m_{Q}^{2}}$ enhancement at $\mathcal{O}\left(\alpha_{s}^{4}\right)$ (next-toleading order, NLO) compared to $\mathcal{O}\left(\alpha_{s}^{3}\right)$ (LO). This enhancement spoils the perturbative convergence in $\alpha_{s}$, shedding light on another possible enhancement from $\mathcal{O}\left(\alpha_{s}^{5}\right)$ (next-tonext-to-leading order, NNLO) corrections, while the accomplishment of the full NNLO calculation is even lacking today. The sole reason is the partonic cross sections $\frac{d \hat{\sigma}}{d P_{T}^{2}}$, before convoluting PDFs, are asymptotically scaling as $\left(\frac{2 m_{Q}}{P_{T}}\right)^{4} \frac{1}{P_{T}^{4}}$ (next-to-next-to-leading power, $\mathrm{NNLP}),\left(\frac{2 m_{Q}}{P_{T}}\right)^{2} \frac{1}{P_{T}^{4}}$ (next-to-leading power, NLP) and $\frac{1}{P_{T}^{4}}$ (leading power, LP) at LO, NLO, 


\begin{tabular}{|c|c|c|c|c|c|c|}
\hline \multirow{2}{*}{ Accuracy } & \multicolumn{2}{|c|}{${ }^{3} S_{1}^{[1]}$} & \multicolumn{2}{c|}{${ }^{3} S_{1}^{[8]}$} & \multicolumn{2}{c|}{${ }^{1} S_{0}^{[1,8]},{ }^{3} P_{J}^{[1,8]},{ }^{1} P_{1}^{[1,8]}$} \\
\cline { 2 - 7 } & LP & NLP & LP & NLP & LP & NLP \\
\hline LO & $\alpha_{s}^{5}$ & $\alpha_{s}^{4}$ & $\alpha_{s}^{3}$ & $\alpha_{s}^{3}$ & $\alpha_{s}^{4}$ & $\alpha_{s}^{3}$ \\
\hline NLO & $\alpha_{s}^{6}$ & $\alpha_{s}^{5}$ & $\alpha_{s}^{4}$ & $\alpha_{s}^{4}$ & $\alpha_{s}^{5}$ & $\alpha_{s}^{4}$ \\
\hline
\end{tabular}

Table 2. The first $\alpha_{s}$ orders needed in the SDCs for both LP and NLP in $P_{T}$ of various Fock states in their hadroproduction in order to achieve the LO and NLO QCD accuracies.

NNLO respectively. ${ }^{1}$ Therefore, even with a full NNLO calculation at $\mathcal{O}\left(\alpha_{s}^{5}\right)$, the accuracy for the LP part of ${ }^{3} S_{1}^{[1]}$ hadroproduction is still at LO level, while the NLP piece is indeed NLO accurate. A NLO accuracy of the LP contribution can only be achieved with a nextto-NNLO calculation in $\alpha_{s}$ for the SDC. The situation is slightly better though still similar for the other Fock states listed in table 1 . Like ${ }^{1} S_{0}^{[1,8]},{ }^{3} P_{J}^{[1,8]},{ }^{1} P_{1}^{[1,8]}$, the NLP (LP) parts of SDCs appear firstly at LO (NLO) in $\alpha_{s}$. On the other hand, because of the same quantum number as the gluon, ${ }^{3} S_{1}^{[8]}$ has the leading $P_{T}$ behaviour as the jet, which means the LP channel is already opened at LO $\mathcal{O}\left(\alpha_{s}^{3}\right)$. In table 2 , we have collected the first $\alpha_{s}$ powers in order to achieve the LO and NLO QCD accuracies for various Fock states at both LP and NLP in $P_{T}$.

Following this observation, the complete NLO result for ${ }^{3} S_{1}^{[1]}$ production is possible to be reproduced by the tree-level matrix element alone at $\mathcal{O}\left(\alpha_{s}^{4}\right)$ after introducing an ad hoc infrared cutoff. A first attempt was given in ref. [3] to introduce an invariant-mass cut on any final-final and initial-final massless parton pairs, which was called NLO ${ }^{\star}$. It can successfully reproduce the high- $P_{T}$ NLO calculation for ${ }^{3} S_{1}^{[1]}$ production. ${ }^{2}$ The same infrared cut can be imposed in the phase-space integration of the $\mathcal{O}\left(\alpha_{s}^{5}\right)$ tree-level matrix element. Another giant $\mathrm{K}$ factor was observed compared to the NLO calculation at high $P_{T}$, which may question on the extractions of color-octet LDMEs based on NLO calculations [6-10]. In contrast, a suspicion in ref. [11] on the size of $\mathcal{O}\left(\alpha_{s}^{5}\right)$ was given from their $P_{T}$ scaling reanalysis of the $\mathrm{NNLO}^{\star}$ curves. Instead of the $P_{T}$ power enhancement, the observed giant $\mathrm{K}$ factor $\frac{d \sigma^{\mathrm{NNLO}}}{d \sigma^{\mathrm{NLO}}}$ is mainly attributed to the introduction of the infrared cutoff. Therefore, a reliable estimate of the size of $\mathcal{O}\left(\alpha_{s}^{5}\right)$ is still missing. It is necessary to clarify the situation before drawing a solid conclusion.

The aim of this paper is to introduce an infrared-safe method to cure the problematic giant $\mathrm{K}$ factors appearing in the SDC calculations in particular for high- $P_{T}$ quarkonium production without performing complete higher-order calculations. ${ }^{3}$ In contrast to the NLO ${ }^{\star}$

\footnotetext{
${ }^{1}$ Rigorously speaking, the associated production of ${ }^{3} S_{1}^{[1]}$ with the same flavoured heavy quark pair contributes $\mathcal{O}\left(\alpha_{s}^{4}\right)$ and is LP in $P_{T}$. We guide the readers to the discussion on this part in section 4.4.

${ }^{2}$ Besides the single ${ }^{3} S_{1}^{[1]}$ production, NLO ${ }^{\star}$ cut was also applied to ${ }^{3} S_{1}^{[1]}+{ }^{3} S_{1}^{[1]}$ hadroproduction in ref. [4]. $\mathrm{NLO}^{\star}$ calculation is able to well reproduce the complete NLO result [5] in the double charmonium/bottomonium production. Its good performance may rely on the fact that like the single ${ }^{3} S_{1}^{[1]}$ production, the LO SDC of ${ }^{3} S_{1}^{[1]}+{ }^{3} S_{1}^{[1]}$ is also NNLP in $P_{T}$ in the large transverse momentum region.

${ }^{3}$ In the processes of elementary particle production, a few proposals to cure the giant $\mathrm{K}$ factors, which are mainly from logarithmic terms in perturbative calculations, are present [12-28]. Unfortunately, none of them is straightforwardly applicable to the power-enhanced contributions in quarkonium production.
} 
calculations, the new method will not introduce the logarithmic dependence from the infrared cutoff. The estimate of the missing higher orders is to use the conventional renormalization and factorization scale variations. It is complemented with the fragmentation function approach, which requires the analytical calculations of different single- and doubleparton fragmentation functions for single and multiple quarkonium production. Another nontrivial task to use the fragmentation function approach is to solve the corresponding coupled evolution equations. It has been shown in ref. [29] that the fragmentation function approach without scale evolution can reproduce the spin-summed NLO cross sections of ${ }^{3} S_{1}^{[1]},{ }^{3} S_{1}^{[8]},{ }^{1} S_{0}^{[8]},{ }^{3} P_{J}^{[8]}$ at high $P_{T}$, which shows the necessity of taking into account both the single-parton (at LP) and the double-parton (at NLP) fragmentation contributions. The factorization theorem for the single-inclusive quarkonium production cross sections in terms of single- and double-parton fragmentation functions was first proven in ref. [30] under the assumption of perturbative QCD factorization.

There are also other appealing reasons to introduce such a method. First of all, it can be used to stabilize the higher-order QCD corrections in quarkonium associated production processes, where most of them are absent of complete NLO calculations. The possible cancellations between S-wave and P-wave are guaranteed in our approach. For instance, in the double $J / \psi$ at the LHC, it requires a NNLO calculation to have the full cancellations between S-wave and P-wave Fock states. As we will see later in this paper, the good reproduction of the NLO results both in the spin-summed and spin-dependent cross sections for single quarkonium production at high $P_{T}$ can serve as a fast way to the future phenomenology studies. In practice, the phenomenology from a complicated calculation scales as an inverse power of the computation time.

The outline of the remaining context is following. After introducing the remainders of P-wave counterterms in section 2, we will show that one can reproduce the NLO results for most of the Fock states (except ${ }^{3} S_{1}^{[8]}$ ) with fairly simple cuts based on tree-level matrix elements in section 3. These simple cuts are not sufficient to remove large logarithms introduced by the phase space cut parameters. Hence, a general infrared-safe method is introduced to obtain the giant $\mathrm{K}$ factors for all the Fock states relevant for $J / \psi$ and $\chi_{c J}$ production in section 4. Finally, we draw our conclusions in section 5. An instruction on how to use HELAC-ONIA [31, 32] to perform the calculations done in this paper is given in appendix A. The appendix B contains supplemental figures.

\section{Remainders of $\mathrm{P}$-wave counterterms}

It is well-known that the remaining infrared divergences in the SDC computations for the productions and decays of $\mathrm{P}$-wave Fock states should be cancelled by the P-wave counterterms arising from the renormalization group running of S-wave LDMEs beyond LO in $\alpha_{s}$, which is analogous to the remaining collinear divergences absorbed by the PDF counterterms in a peturbative QCD calculation. The renormalization of NRQCD operators links the S-wave LDMEs with the P-wave LDMEs as shown in eq. (150) of ref. [33]. Such counterterms, after cancelling infrared divergences with the real and virtual matrix elements, will leave finite remainders proportional to the S-wave SDCs and P-wave LDMEs. The 
introduction of the $\mathrm{P}$-wave counterterms is crucial especially in the case that the $\mathrm{S}$-wave SDCs are much larger than the P-wave SDCs. In particular, the negative P-wave SDCs for heavy quarkonium hadroproduction at high $P_{T}$ could be attributed to these negative remainders. We have implemented the following finite remainders of $\mathrm{P}$-wave counterterms:

$$
\begin{aligned}
d \sigma^{\mathcal{C}}\left({ }^{3} P_{J}^{[8]}\right)= & d \hat{\sigma}^{\text {Born }}\left({ }^{3} S_{1}^{[1]}\right) \times\left(\frac{4}{3} \frac{\alpha_{s}}{\pi} \frac{\log \frac{m_{Q}^{2}}{4 \mu_{\Lambda}^{2}}}{m_{Q}^{2}}\right) \times\left\langle\mathcal{O}\left({ }^{3} P_{J}^{[8]}\right)\right\rangle \\
& +d \hat{\sigma}^{\text {Born }}\left({ }^{3} S_{1}^{[8]}\right) \times\left(\frac{5}{9} \frac{\alpha_{s}}{\pi} \frac{\log \frac{m_{Q}^{2}}{4 \mu_{\Lambda}^{2}}}{m_{Q}^{2}}\right) \times\left\langle\mathcal{O}\left({ }^{3} P_{J}^{[8]}\right)\right\rangle, \\
d \sigma^{\mathcal{C}}\left({ }^{3} P_{J}^{[1]}\right)= & d \hat{\sigma}^{\text {Born }}\left({ }^{3} S_{1}^{[8]}\right) \times\left(\frac{8}{27} \frac{\alpha_{s}}{\pi} \frac{\log \frac{m_{Q}^{2}}{4 \mu_{\Lambda}^{2}}}{m_{Q}^{2}}\right) \times\left\langle\mathcal{O}\left({ }^{3} P_{J}^{[1]}\right)\right\rangle, \\
d \sigma^{\mathcal{C}}\left({ }^{1} P_{1}^{[8]}\right)= & d \hat{\sigma}^{\text {Born }}\left({ }^{1} S_{0}^{[1]}\right) \times\left(\frac{4}{3} \frac{\alpha_{s}}{\pi} \frac{\log \frac{m_{Q}^{2}}{4 \mu_{\Lambda}^{2}}}{m_{Q}^{2}}\right) \times\left\langle\mathcal{O}\left({ }^{1} P_{1}^{[8]}\right)\right\rangle \\
& +d \hat{\sigma}^{\text {Born }}\left({ }^{1} S_{0}^{[8]}\right) \times\left(\frac{5}{9} \frac{\alpha_{s}}{\pi} \frac{\log \frac{m_{Q}^{2}}{4 \mu_{\Lambda}^{2}}}{m_{Q}^{2}}\right) \times\left\langle\mathcal{O}\left({ }^{1} P_{1}^{[8]}\right),\right. \\
d \sigma^{\mathcal{C}}\left({ }^{1} P_{1}^{[1]}\right)= & d \hat{\sigma}^{\text {Born }}\left({ }^{1} S_{0}^{[8]}\right) \times\left(\frac{8}{27} \frac{\alpha_{s}}{\pi} \frac{\log \frac{m_{Q}^{2}}{4 \mu_{\Lambda}^{2}}}{m_{Q}^{2}}\right) \times\left\langle\mathcal{O}\left({ }^{1} P_{1}^{[1]}\right)\right\rangle,
\end{aligned}
$$

where $m_{Q}$ is the mass of the heavy quark and $\mu_{\Lambda}$ is the NRQCD scale. In the following, we will set $\mu_{\Lambda}=m_{Q}$ as usually done in the complete NLO calculations. These remainders have already been implemented in the HELAC-ONIA [31, 32]. They are necessary ingredients to reproduce the NLO results, which we will show in the following two sections.

\section{$3 \quad$ A first step towards NLO}

From the discussion in the section 1, it is known that large NLO QCD corrections to the $J / \psi$ production at a high-energy hadron collider are mainly due to the emergence of new $P_{T}$ power-enhanced fragmentation contributions. Hence, all S- and P-wave Fock states except ${ }^{3} S_{1}^{[8]}$ receive giant $\mathrm{K}$ factors from NLO QCD calculations.

We first introduce the following basic phase space cuts in order to take into account the hard radiations without using virtual amplitudes. In real part at $\mathcal{O}\left(\alpha_{s}^{4}\right)$, exact 2 lightflavoured jets ${ }^{4}$ satisfying $P_{T}(j)>P_{T}^{\min }$ and $|y(j)|<y^{\max }$ are required, which is denoted as $d \sigma^{\mathcal{R}_{0}}$. The phase space integrations of Born $d \sigma^{\mathcal{B}}\left(\mathcal{O}\left(\alpha_{s}^{3}\right)\right)$ and the remainders of the NRQCD P-wave counterterms $d \sigma^{\mathcal{C}}\left(\mathcal{O}\left(\alpha_{s}^{4}\right)\right)$ are infrared safe with $P_{T}$ (onium) larger than

\footnotetext{
${ }^{4}$ We mean "light-flavoured jet" here as a cluster of gluon, up, down, strange (anti-)quarks. Similarly, the light-flavoured partons are defined as gluon, up, down, strange (anti-)quarks.
} 


\begin{tabular}{|c|c|c|c|c|}
\hline$\left\langle\mathcal{O}\left({ }^{3} S_{1}^{[1]}\right)\right\rangle$ & $\left\langle\mathcal{O}\left({ }^{3} S_{1}^{[8]}\right)\right\rangle$ & $\left\langle\mathcal{O}\left({ }^{1} S_{0}^{[8]}\right)\right\rangle$ & $\left\langle\mathcal{O}\left({ }^{3} P_{J}^{[8]}\right)\right\rangle /(2 J+1)$ & $\left\langle\mathcal{O}\left({ }^{3} P_{J}^{[1]}\right)\right\rangle /(2 J+1)$ \\
\hline $1.16 \mathrm{GeV}^{3}$ & $9.03 \cdot 10^{-3} \mathrm{GeV}^{3}$ & $1.46 \cdot 10^{-2} \mathrm{GeV}^{3}$ & $3.43 \cdot 10^{-2} \mathrm{GeV}^{5}$ & $0.107 \mathrm{GeV}^{5}$ \\
\hline
\end{tabular}

Table 3. The values of LDMEs used in the differential distributions of various Fock states.

a given positive value $P_{T}^{\min }$ (onium). We call the summed results of $d \sigma^{\mathcal{B}}+d \sigma^{\mathcal{R}_{0}}+d \sigma^{\mathcal{C}}$ as approximated NLO (aNLO).

In the following, we take $P_{T}^{\min }$ (onium) $=5 \mathrm{GeV}$, and light-flavoured jets are clustered with anti- $k_{T}$ algorithm [34] using radius $R=0.5$ and $|y(j)|<5, P_{T}(j)>P_{T}^{\min }$ by FASTJET [35]. We will vary $P_{T}^{\min }$ from $3 \mathrm{GeV}$ to $6 \mathrm{GeV}$ as a way to estimate the infrared-cut dependence. We have shown the spin-summed double differential distributions for the $c \bar{c}$ Fock state ${ }^{3} S_{1}^{[1]}$ in figure 1 with the $13 \mathrm{TeV}$ proton-proton collisions, while the distributions for the 5 Fock states ${ }^{1} S_{0}^{[8]},{ }^{3} P_{J}^{[8]},{ }^{3} P_{0}^{[1]},{ }^{3} P_{1}^{[1]},{ }^{3} P_{2}^{[1]}$ are displayed in figure 18 as our supplemental material. The complete NLO curves (denoting as NLO) from refs. [7, 36] are also shown in order to have a comparison. The red-hatched bands represent the infrared cut variations $P_{T}^{\min } \in[3,6] \mathrm{GeV}$, and the grey bands are the uncertainty from the independent variations of renormalization and factorization scales $\mu_{R}, \mu_{F}$ around the central value $\mu_{0}=\sqrt{P_{T}^{2}(\mathrm{onium})+4 m_{c}^{2}}$ by a factor of 2 . It is interesting to notice that the scale uncertainty in general captures the missing virtual and soft/collinear pieces. The agreements between NLO and aNLO are improved as $P_{T}$ (onium) increases. A similar behaviour can be observed for the spin-dependent differential cross sections shown in figure 2 for ${ }^{3} S_{1}^{[1]}$ and in figures 19, 20 for ${ }^{3} P_{J}^{[8]},{ }^{3} P_{1}^{[1]},{ }^{3} P_{2}^{[1]}$ Fock states, where the NLO curves are from refs. [8, 37]. The spin-density matrix elements of the scalars ${ }^{1} S_{0}^{[8]},{ }^{3} P_{0}^{[1]}$ are trivial. ${ }^{5}$ We have utilized CTEQ6M PDF [38] to be consistent with the NLO results. For the reproducible purpose, the values of LDMEs for the distributions of the Fock states are listed in table 3.

Because the LP in $P_{T}$ for ${ }^{3} S_{1}^{[8]}$ already exists at Born $d \sigma^{\mathcal{B}}$ (i.e. $\mathcal{O}\left(\alpha_{s}^{3}\right)$ ) from the gluon fragmentation, it is expected that the scale uncertainty at $\mathrm{LO}$ would already give a reliable estimate of the missing NLO QCD corrections, which is indeed observed from the leftpanel of figure 3. In such a case, a request of 2 light-flavoured jets in the computation of $d \sigma^{\mathcal{R}_{0}}$ is insufficient to obtain an infrared-safe differential cross section. From the rightpanel of figure 3 , the aNLO $P_{T}$ spectra are too hard compared to the complete NLO ones. The reason is because of the large logarithms arising from the very asymmetric dijet system $P_{T}\left(j_{1}\right) \gg P_{T}\left(j_{2}\right)$. Such a configuration is suppressed in other Fock states, because the leading fragmentation topologies require at least one light-flavoured parton along with the quarkonium direction at high $P_{T}$. The weights of the asymmetric dijet events will be enhanced due to the unphysical logarithm $\log \frac{P_{T}\left(j_{1}\right)}{P_{T}\left(j_{2}\right)}$ in the aNLO calculations of ${ }^{3} S_{1}^{[8]}$, which should be in principle cancelled by the virtual contributions because of the unitarity. Therefore, one must introduce a more general infrared-safe method to avoid these large logarithms, and at meantime one should maintain the hard radiations from the real contributions.

\footnotetext{
${ }^{5}$ The spin-density matrix elements $\frac{d \sigma_{J_{z}} J_{z}}{d P_{T}}$ shown in this paper are defined in the usual helicity frame.
} 


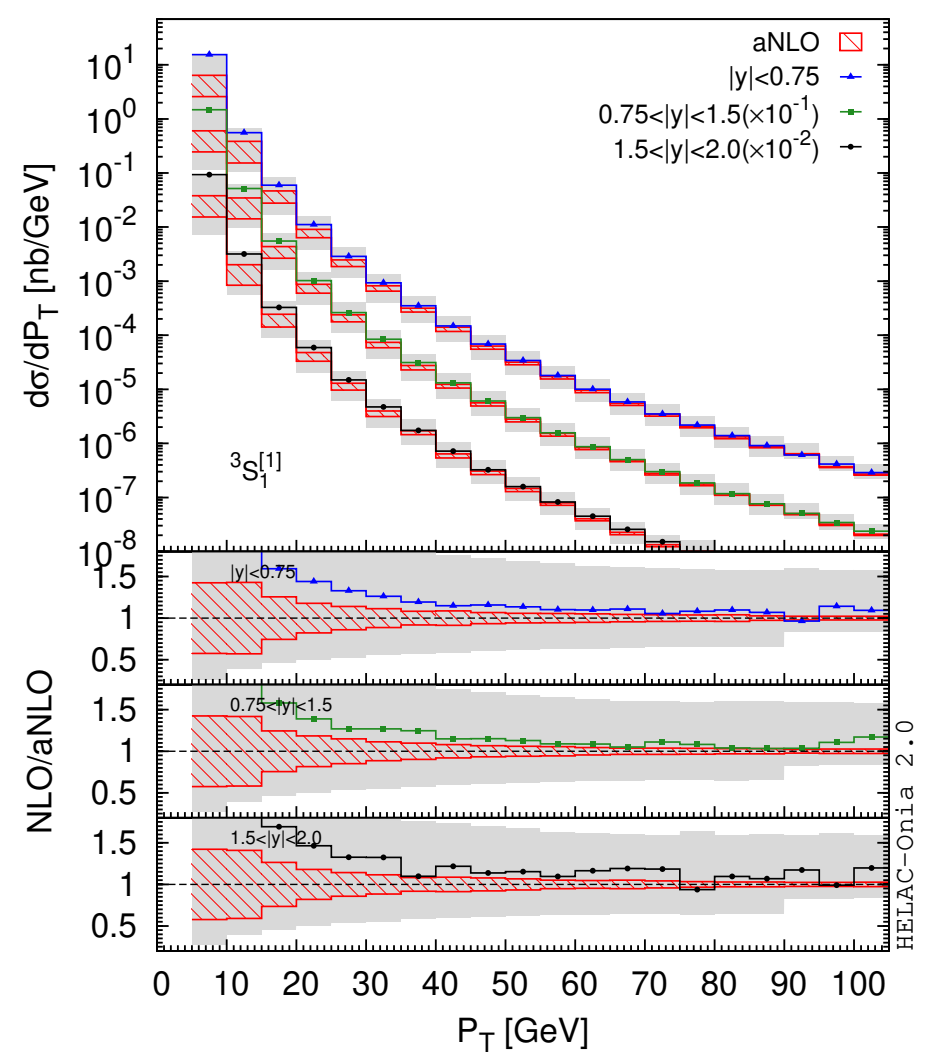

Figure 1. Comparison of spin-summed differential cross sections for the Fock state ${ }^{3} S_{1}^{[1]}$ between our aNLO calculations and the complete NLO calculations.
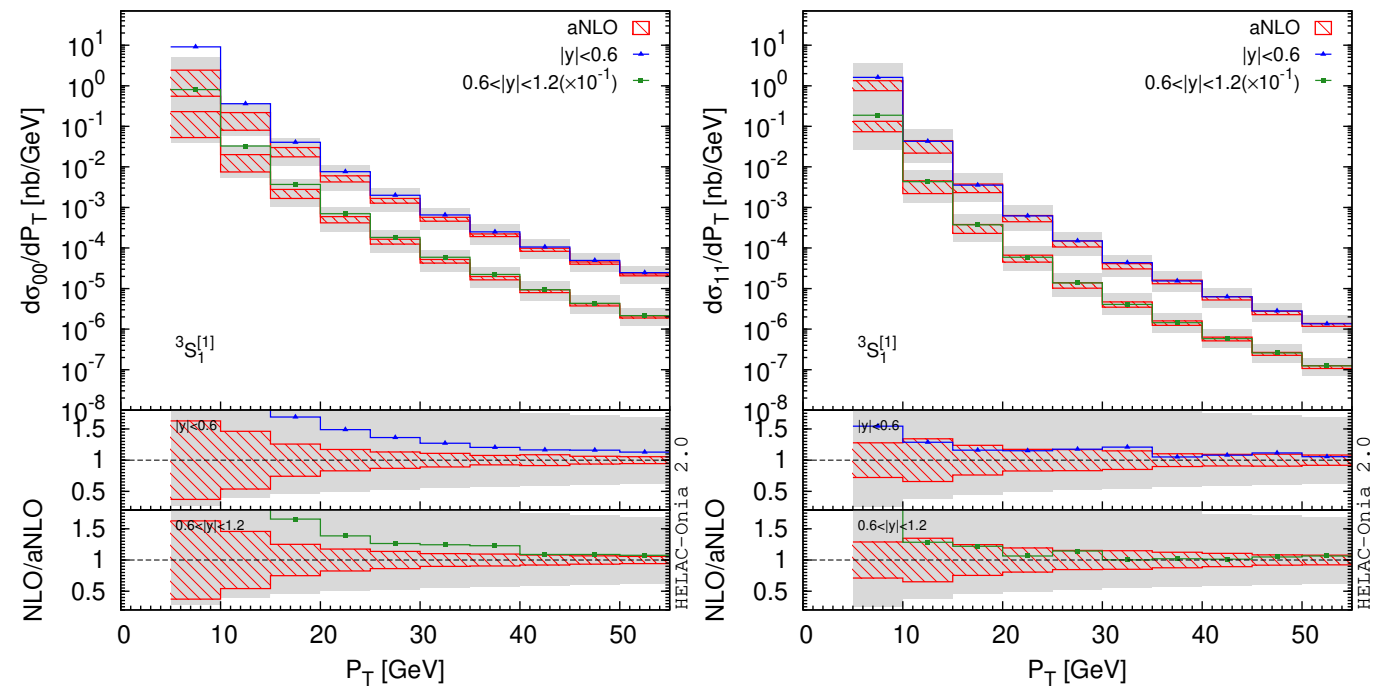

Figure 2. Comparisons of spin-dependent differential cross sections for the Fock state ${ }^{3} S_{1}^{[1]}$ between our aNLO calculations and the complete NLO calculations. 

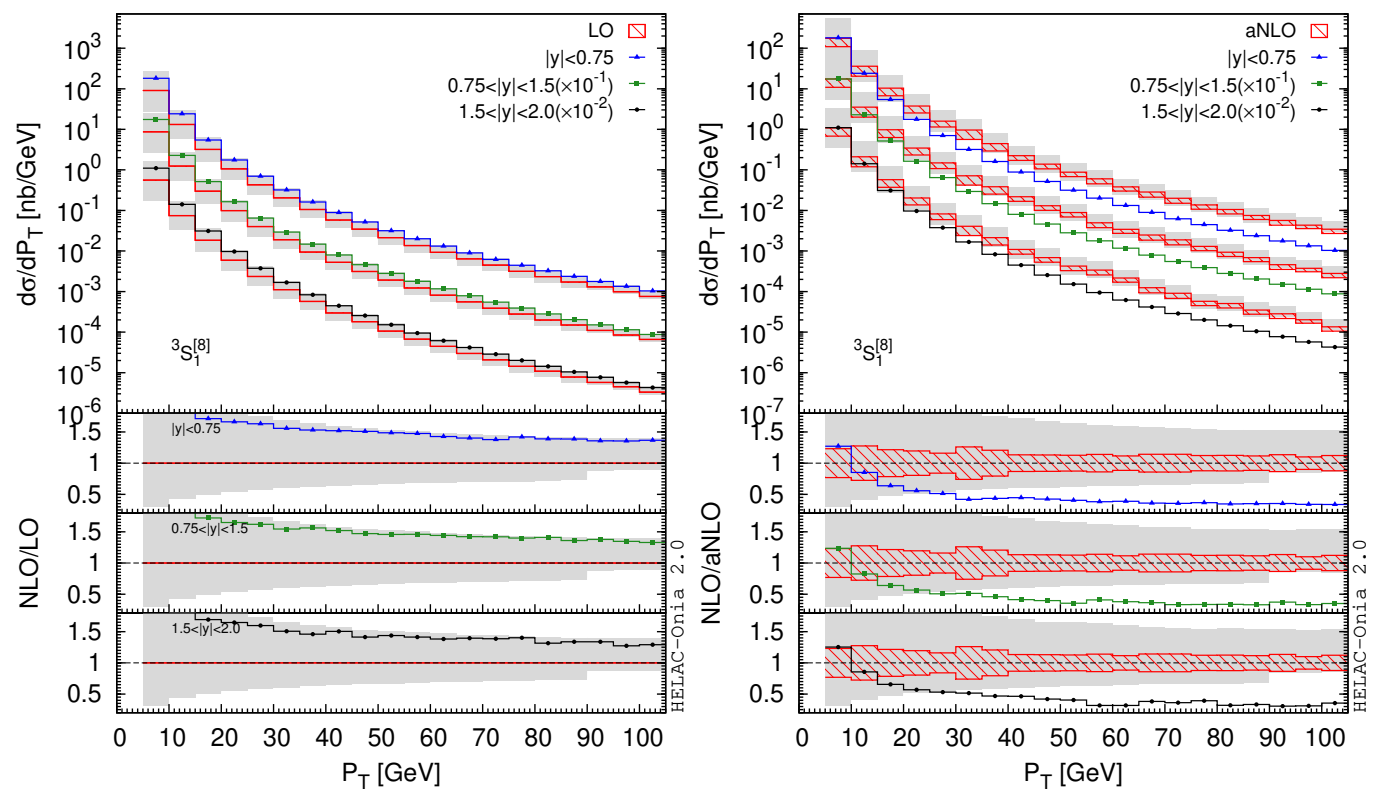

Figure 3. Comparisons of spin-summed differential cross sections for the Fock state ${ }^{3} S_{1}^{[8]}$ between LO (left), aNLO (right) calculations and the complete NLO calculations.

\section{A general infrared-safe method}

\subsection{Infrared-safe cutoffs}

Let us assume a LO fragmentation process for a given Fock state $\mathcal{O}_{n}$ is accompanying with $k$ final massless partons:

$$
\mathcal{F}: p_{0} \rightarrow P_{\mathcal{O}_{n}}+p_{1}+\cdots+p_{k}
$$

For a given observable, one needs to consider $\mathcal{O}_{n}$ plus $i$ recoiling partons. For example, in the case of the transverse-momentum distribution for a single quarkonium production (schematically depicted in figure 4), the quarkonium at least recoils against one parton at the lowest order (bar the zero transverse momentum bin). The soft- and collinear-safe calculations can be achieved based on pure tree-level matrix elements via the following conditions:

1. The number of jets is larger than $i+1$ with the transverse momentum of jet $P_{T}(j)>$ $P_{T}^{\min }$ and the rapidity $|y(j)|<y^{\max } . \mathcal{O}_{n}$ is also taken into account in the jet-clustering procedure. One should make sure that there is exactly one jet containing $\mathcal{O}_{n}$ passing the above $P_{T}$ and rapidity cuts. Such a jet is called an onium-jet here.

2. In the onium-jet, there are at least $k$ light-flavoured partons to fulfill the above fragmentation process. Let us say there are $m$ light-flavoured partons inside the onium-jet with $m \geq k{ }^{6}$

\footnotetext{
${ }^{6}$ At LO one should have $m=k$ since the configuration of $i+k-m<i$ recoiling partons is zero by definition for the given observable when $m>k$.
} 

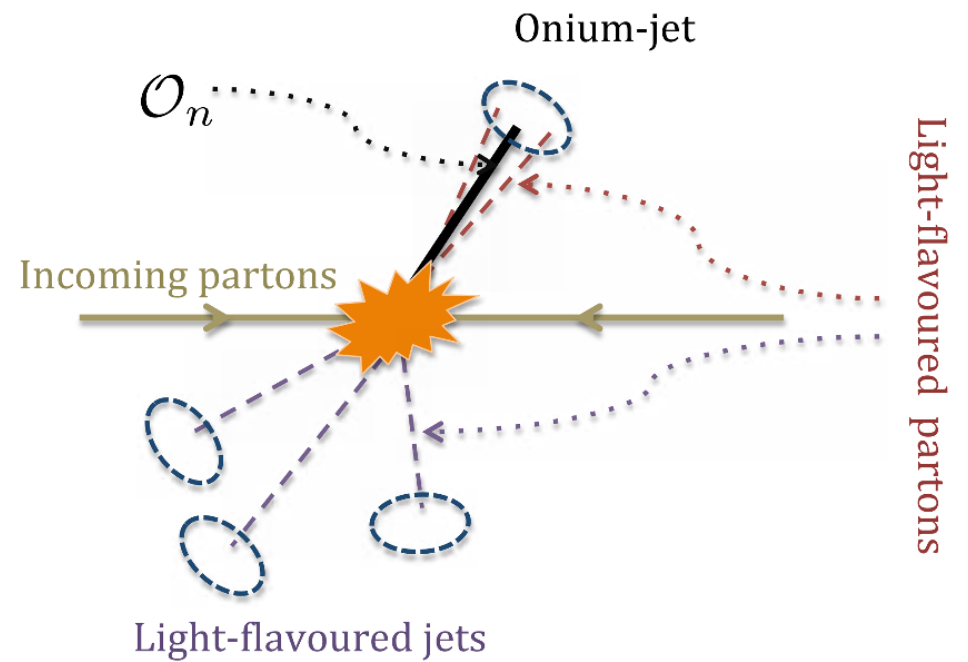

Figure 4. Schematic depiction of inclusive quarkonium $\mathcal{O}_{n}$ production.

3. If $m \geq 2$, each pair of parton 1 and 2 inside the onium-jet should pass the following soft drop condition [39]

$$
\frac{\min \left(p_{T, 1}, p_{T, 2}\right)}{p_{T, 1}+p_{T, 2}}>z_{\text {cut }}\left(\frac{\Delta R_{12}}{R_{0}}\right)^{\beta}
$$

where $p_{T, i}$ is the transverse momentum of parton $i$ and $\Delta R_{12}=\sqrt{\Delta \phi_{12}^{2}+\Delta y_{12}^{2}}$. The above cut already excludes the soft singularity as long as $z_{\text {cut }}>0$, while the requirement of the collinear safety is guaranteed by choosing $\beta<0 . R_{0}$ is the original jet radius, which is an order one number.

The condition eq. (4.2) in item 3 is chosen to kill the infrared unsafe configurations (a) and (b) given in figure 5. Either when partons 1 and 2 are close to be collinear $\Delta R_{12} \ll R_{0}$ or if one parton is soft $p_{T, 2} \ll p_{T, 1}$, eq. (4.2) cannot be fulfilled when $z_{\text {cut }}>0, \beta<0$. In practice, the absolute value of $\beta$ is at order one and $z_{\text {cut }}$ is at the order of $v^{2}$.

If one goes to extra $o$ radiations (i.e. $\mathcal{O}_{n}$ plus $i+k+o$ final light-flavoured QCD partons), one should impose the following additional cuts:

4. There are $i+k+o-m$ partons outside the onium-jet. Each parton should form a single jet within $P_{T}(j)>P_{T}^{\min }$ and $|y(j)|<y^{\max }$ to avoid the collinear divergences. In order to get rid of large logarithms from infrared cuts arising from the soft largeangle radiations illustrated in the case (d) in figure 5 , when $i+k+o-m \geq 2$, it is necessary to impose an asymmetric cut on these parton jets

$$
\frac{\min \left(P_{T}\left(j_{1}\right), \cdots, P_{T}\left(j_{i+k+o-m}\right)\right)}{\max \left(P_{T}\left(j_{1}\right), \cdots, P_{T}\left(j_{i+k+o-m}\right)\right)}>z_{\mathrm{cut}, \mathrm{a}}
$$

The value of $z_{\text {cut,a }}$ should be a positive number smaller than 1 but not close to 0 . It is important to vary its value in order to assess this cut dependence. 


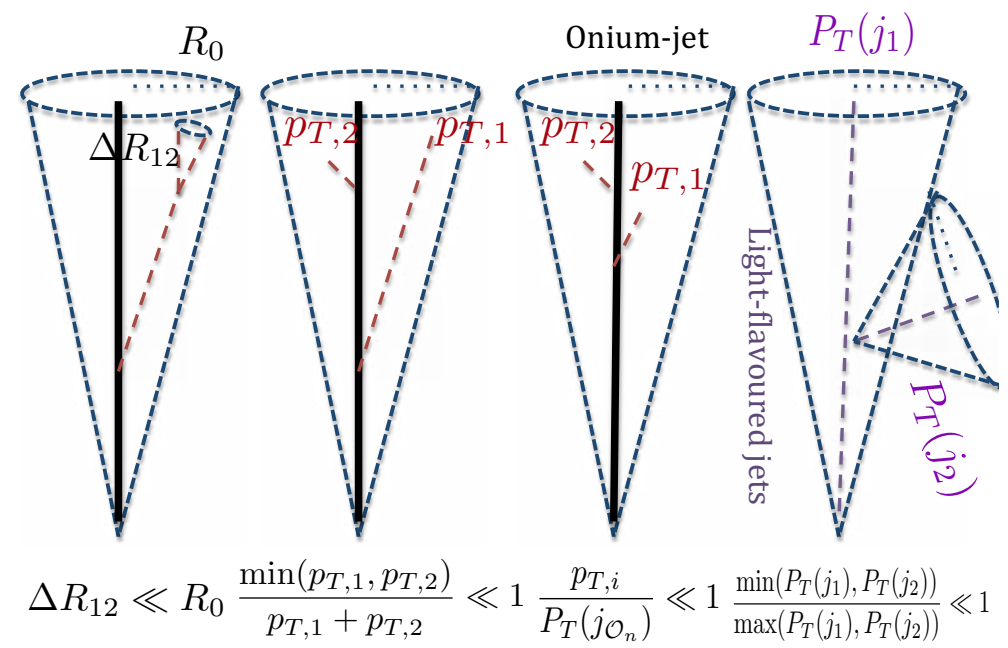

(a)

(b)

(c)

(d)

Figure 5. Infrared unsafe configurations to be considered in inclusive quarkonium production, where the first 3 subfigures are for the onium jet $j_{\mathcal{O}_{n}}$ and the last one is for the light-flavoured jets.

5. If $k=0,{ }^{7}$ and $m>0$, each parton 1 in the onium jet $j_{\mathcal{O}_{n}}$ should pass the soft cut

$$
z_{1}>z_{\text {cut }, \mathrm{s}}
$$

where $z_{1}$ can be the energy fraction $\frac{E_{1}}{E\left(j_{\mathcal{O}_{n}}\right)}$, the transverse mass fraction $\frac{p_{T, 1}}{\sqrt{P_{T}^{2}\left(j_{\mathcal{O}_{n}}\right)+M^{2}\left(j_{\left.\mathcal{O}_{n}\right)}\right.}}$, the transverse momentum fraction $\frac{p_{T, 1}}{P_{T}\left(j_{\mathcal{O}_{n}}\right)}$ or other similar fractions corresponding to $z_{1} \rightarrow 0$ when the parton 1 is soft. This condition is needed in order to kill the case (c) in figure 5, where all light-flavoured partons in the onium-jet $j_{\mathcal{O}_{n}}$ can be soft and the condition eq. (4.2) is still satisfied. Similar to the value of $z_{\text {cut }}$ in eq. (4.2), the proper value of $z_{\text {cut,s }}$ should be $\mathcal{O}\left(v^{2}\right)$ as the effect of the soft radiations should be absorbed into the long-distance part of the quarkonium.

In fact, the combination of items 1-5 introduces a general infrared-safe method for any Fock state production if $k=0$ is assumed at the beginning. ${ }^{8}$ In other words, we do not need to pay a special attention to which kind of fragmentation process $\mathcal{F}$ is allowed for a given Fock state. We call such cuts as STOP cuts, where "STOP" is an acronym of "STabilize quarkOnium Production".

In the case of the $P_{T}$ spectrum of a quarkonium $\mathcal{O}_{n}$ production at a hadron collider, $i$ is equal to 1 and the LO process is $\mathcal{O}_{n}$ plus one parton. For a real emission process $\mathcal{O}_{n}$ plus $o+1$ partons with $o>0$, we should impose the cuts listed in items $1-5$ with $k=0$, where the condition in item 2 is fulfilled automatically. Same as the previous section, we will denote the Born contribution at $\mathcal{O}\left(\alpha_{s}^{3}\right)$ as $d \sigma^{\mathcal{B}}$ and the remainders of the $\mathrm{P}$-wave counterterms at $\mathcal{O}\left(\alpha_{s}^{4}\right)$ as $d \sigma^{\mathcal{C}} \cdot d \sigma^{\mathcal{R}_{\mathrm{STOP}}}\left(d \sigma^{\mathcal{R}_{\mathrm{STOP}}^{2}}\right)$ stands for the contribution from $\mathcal{O}_{n}$ plus two (three) partons within the STOP cuts.

\footnotetext{
${ }^{7}$ For example, $\mathcal{O}_{n}=Q \bar{Q}\left({ }^{3} S_{1}^{[8]}\right)$ has $g \rightarrow Q \bar{Q}\left({ }^{3} S_{1}^{[8]}\right)$ fragmentation at LO.

${ }^{8}$ When $k=0$, the cut in item 2 will not be applied.
} 


\subsection{Reproducing NLO results}

In this section, we will present the results up to NLO QCD corrections (i.e. $\mathcal{O}\left(\alpha_{s}^{4}\right)$ ). In order to differentiate our partial NLO calculations with the complete NLO results, we will denote our partial NLO calculations by imposing STOP cuts as "nLO", i.e. $d \sigma^{\mathrm{nLO}} \equiv$ $d \sigma^{\mathcal{B}}+d \sigma^{\mathcal{C}}+d \sigma^{\mathcal{R}_{\text {STOP }}}$. In the following, we will illustrate that the complete NLO results can be reproduced with the tree-level generators under the following setup of the STOP cuts:

$$
\begin{aligned}
P_{T}(j) & >P_{T}^{\min }, P_{T}^{\min } \in[3,6] \mathrm{GeV},|y(j)|<5.0, \\
z_{\text {cut }} & =0.1, \beta=-1, R_{0}=1.0, \\
z_{\text {cut }, \mathrm{a}} & \in[0.1,0.7], z_{\mathrm{cut}, \mathrm{s}}=\frac{0.1}{m},
\end{aligned}
$$

where $m$ is the number of light-flavoured partons inside the onium jet. Jets are reconstructed with the anti- $k_{T}$ clustering algorithm using FASTJET. Since there is no infrared divergence in the Born after imposing $P_{T}$ (onium) $>0$ cut, the STOP cuts will not be applied to the Born and Born-like counterterm events.

\subsubsection{Reproducing ${ }^{3} S_{1}^{[8]}$}

After imposing the STOP cuts on ${ }^{3} S_{1}^{[8]}$, we can reproduce the complete NLO curves within the theoretical uncertainties. They are shown in figure 6 and figure 7 for the spin-summed and spin-dependent differential cross sections respectively. In the left panel of figure 6 and the upper panels of figure 7, we estimate infrared cutoff dependence (the red-hatched bands) via the combined variations of $P_{T}^{\min } \in[3,6] \mathrm{GeV}$ and $z_{\text {cut, } \mathrm{a}} \in[0.1,0.7]$. The grey-shadowed bands represent the scale uncertainties. Opposed to the aNLO results in the right panel of figure 3, it indeed shows that the STOP cuts improve the perturbative calculations, and the transverse-momentum dependence in $\frac{d \sigma^{\mathrm{nLO}}}{d P_{T}}$ is the same as the NLO distributions $\frac{d \sigma^{\mathrm{NLO}}}{d P_{T}}$. It demonstrates that the large logarithmic dependence from the simple cuts in section 3 disappears after imposing the STOP cuts. The STOP-cut dependence (the red-hatched bands) is not reduced by increasing the $P_{T}$ of the quarkonium. It is expected since the LP contribution is already present at LO. The variations of the STOP cut variables only alter the fractions of hard radiations in the real matrix elements, which are not logarithmically enhanced. In fact, a careful tuning of STOP cut parameters can reproduce the NLO results at high precision. In the right panel of figure 6 and the lower panels of figure 7 , we have calculated the ${ }^{3} S_{1}^{[8]}$ differential distributions after using $z_{\text {cut, a }}=0.6$ and $z_{\text {cut }, \mathrm{s}}=\frac{0.2}{m}$. The comparisons to the full NLO calculations imply that the $P_{T}$ spectra of ${ }^{3} S_{1}^{[8]}$ in different rapidity intervals can be precisely reproduced as long as $P_{T}\left({ }^{3} S_{1}^{[8]}\right)>10 \mathrm{GeV}$.

\subsubsection{Reproducing other Fock states}

We are now in the position to check the calculations for the other Fock states with the STOP cuts. Like the case of the simple cuts in section 3, the general infrared-safe STOP cuts can reproduce the complete NLO results within theoretical uncertainties well. These Fock states do not show LP behaviour at LO. The comparisons of nLO calculations to NLO calculations for spin-summed and spin-dependent differential cross sections in the states 

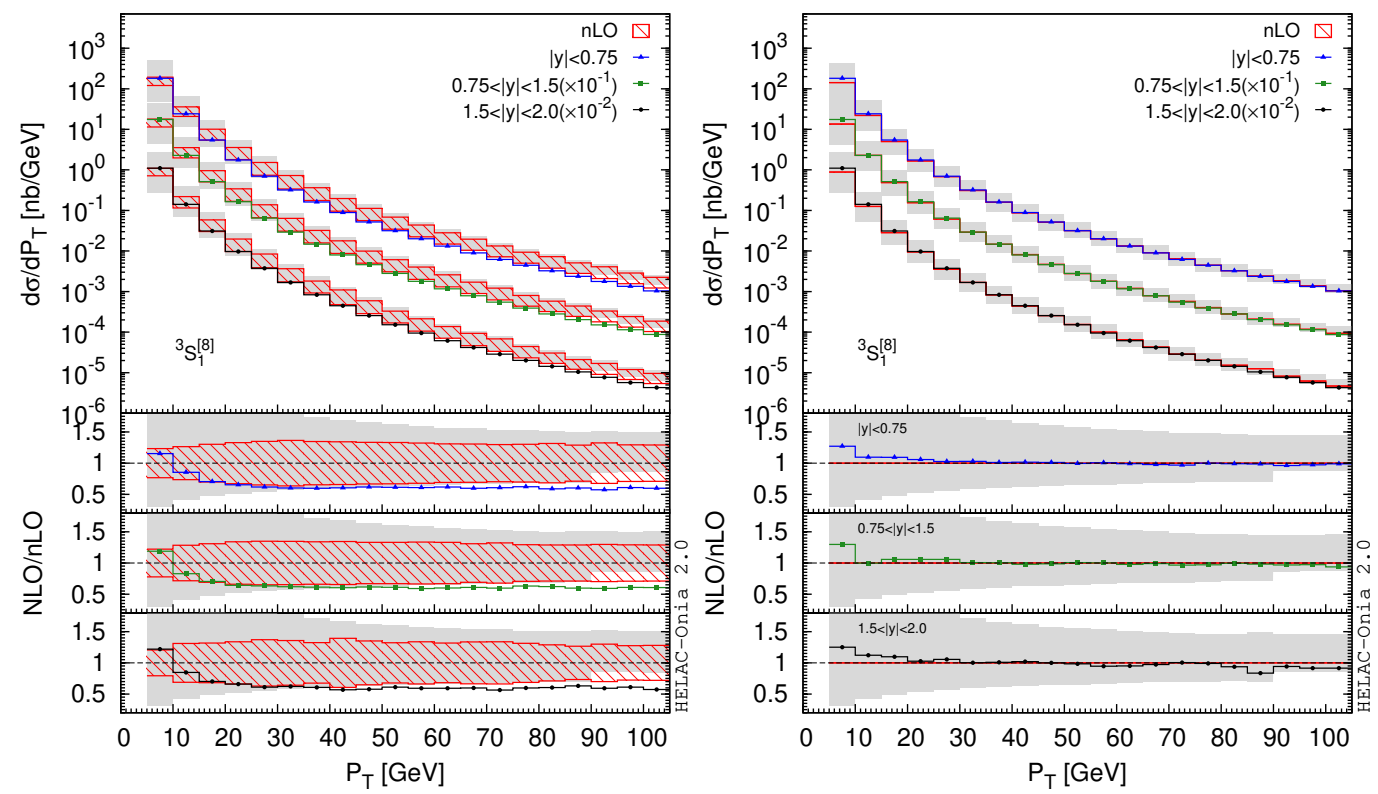

Figure 6. Comparisons of spin-summed differential cross sections for the Fock state ${ }^{3} S_{1}^{[8]}$ between $\mathrm{nLO}$ (left), tunned $\mathrm{nLO}$ with $z_{\text {cut }, \mathrm{a}}=0.6, z_{\mathrm{cut}, \mathrm{s}}=\frac{0.2}{m}$ (right) calculations and the complete NLO calculations.

${ }^{3} S_{1}^{[1]},{ }^{1} S_{0}^{[8]}$ are displayed in figure 8 and figure 9 respectively, while we put the supplemental plots figures 21, 22, 23 for the other Fock states in the appendix B. With the scale variations shown by the grey bands, nLO results in general are able to successfully reproduce the NLO calculations in both cases. The only exception is the differential cross section of ${ }^{1} S_{0}^{[8]}$ (see the right panel of figure 8) at very large $P_{T}$, i.e. $P_{T}>90 \mathrm{GeV}$. Such a discrepancy in ${ }^{1} S_{0}^{[8]}$ can be better understood from the LO fragmentation function $g \rightarrow{ }^{1} S_{0}^{[8]}+g$ [40], which has the functional form

$$
D_{g \rightarrow S_{0}^{[8]}}\left(z_{\text {onium }}\right) \propto 3 z_{\text {onium }}-2 z_{\text {onium }}^{2}+2\left(1-z_{\text {onium }}\right) \log \left(1-z_{\text {onium }}\right)
$$

where $z_{\text {onium }}$ is the momentum fraction of ${ }^{1} S_{0}^{[8]}$. The function peaks at $z_{\text {onium }}=1$. A finite value of $z_{\text {cut,s }}$ in the STOP cuts will remove a non-negligible fraction of radiations in the LP contributions. In fact, we have explicitly checked that if we set $z_{\mathrm{cut}, \mathrm{s}}=\frac{10^{-2}}{\mathrm{~m}}$ instead of $z_{\text {cut }, \mathrm{s}}=\frac{0.1}{m}$, the agreement between nLO and NLO results are significantly improved at large $P_{T}$, which can be found in figure 10. In the spirit of the NRQCD factorization, the soft gluons from the heavy quark pair with the momentum fraction smaller than $v^{2}$ should be absorbed into the LDMEs as well as their energy evolutions, where $v^{2}$ is around 0.3 for the charmonium. Therefore, without taking into account the relativistic corrections, the resolution of NRQCD in describing the heavy quarkonium production should be not better than $v^{2}$. Hence, it is not straightforward to judge which is a better choice between the two different values $z_{\mathrm{cut}, \mathrm{s}}=\frac{0.1}{m}$ and $z_{\mathrm{cut}, \mathrm{s}}=\frac{10^{-2}}{m}$. In fact, we believe $z_{\mathrm{cut}, \mathrm{s}}=\frac{0.1}{m}$ is a compromising choice in order to avoid spoiling the perturbative convergence in the fixed-order calculations by a large $\log$ arithm $\log z_{\mathrm{cut}, \mathrm{s}}$. 

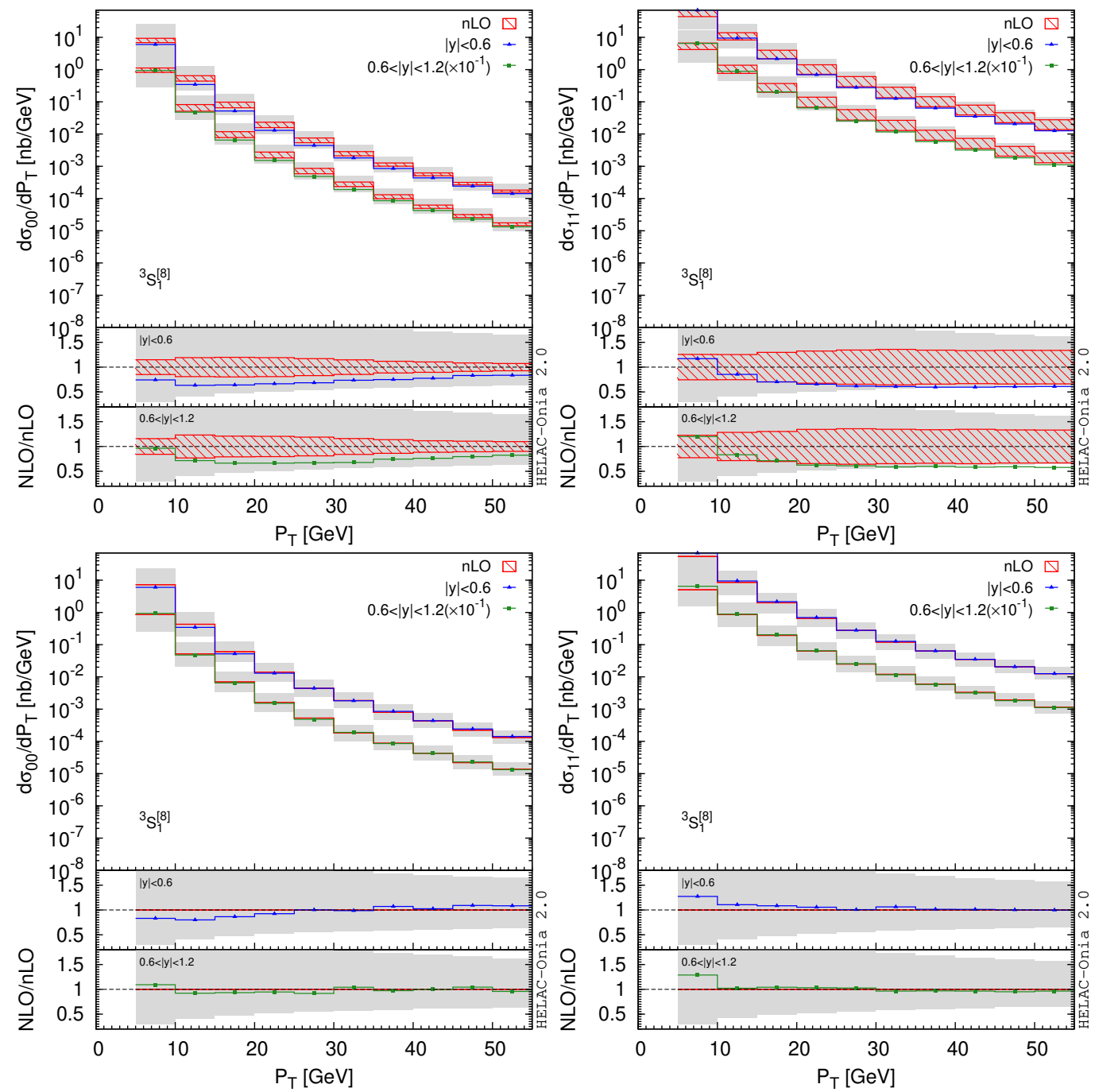

Figure 7. Comparisons of spin-dependent differential cross sections for the Fock state ${ }^{3} S_{1}^{[8]}$ between $\mathrm{nLO}$ (up), tunned $\mathrm{nLO}$ with $z_{\text {cut }, \mathrm{a}}=0.6, z_{\text {cut }, \mathrm{s}}=\frac{0.2}{m}$ (down) calculations and the complete NLO calculations.

We have compared the recent CMS measurement [41] to our nLO calculations (with and without STOP cut tuning on ${ }^{3} S_{1}^{[8]}$ ) for $\psi(2 S)$ production at $13 \mathrm{TeV}$ LHC in figure 11, where the nonperturbative LDMEs are taken from eqs. (2.17) and (2.18) in ref. [42]. A factor $10^{-1}$ has been multiplied to the nLO results with tuned ${ }^{3} S_{1}^{[8]}$ in order to improve the visibility between the two theoretical bands. Without surprising, the CMS data agree very well with our nLO calculations, because nLO does a similarly good job as NLO.

\subsection{Going beyond NLO}

It is usually believed that the color-octet states for $J / \psi$ hadroproduction will not receive giant $\mathrm{K}$ factors beyond NLO as the LP topologies in $P_{T}$ appear at NLO. On the other hand, the color-singlet Fock state ${ }^{3} S_{1}^{[1]}$, which is LO in $v^{2}$ expansion, contains the LP single-gluon fragmentation contributions starting from $\mathrm{NNLO}$ in $\alpha_{s}$ (i.e. $\mathcal{O}\left(\alpha_{s}^{5}\right)$ ). A giant $\mathrm{K}$ factor for 

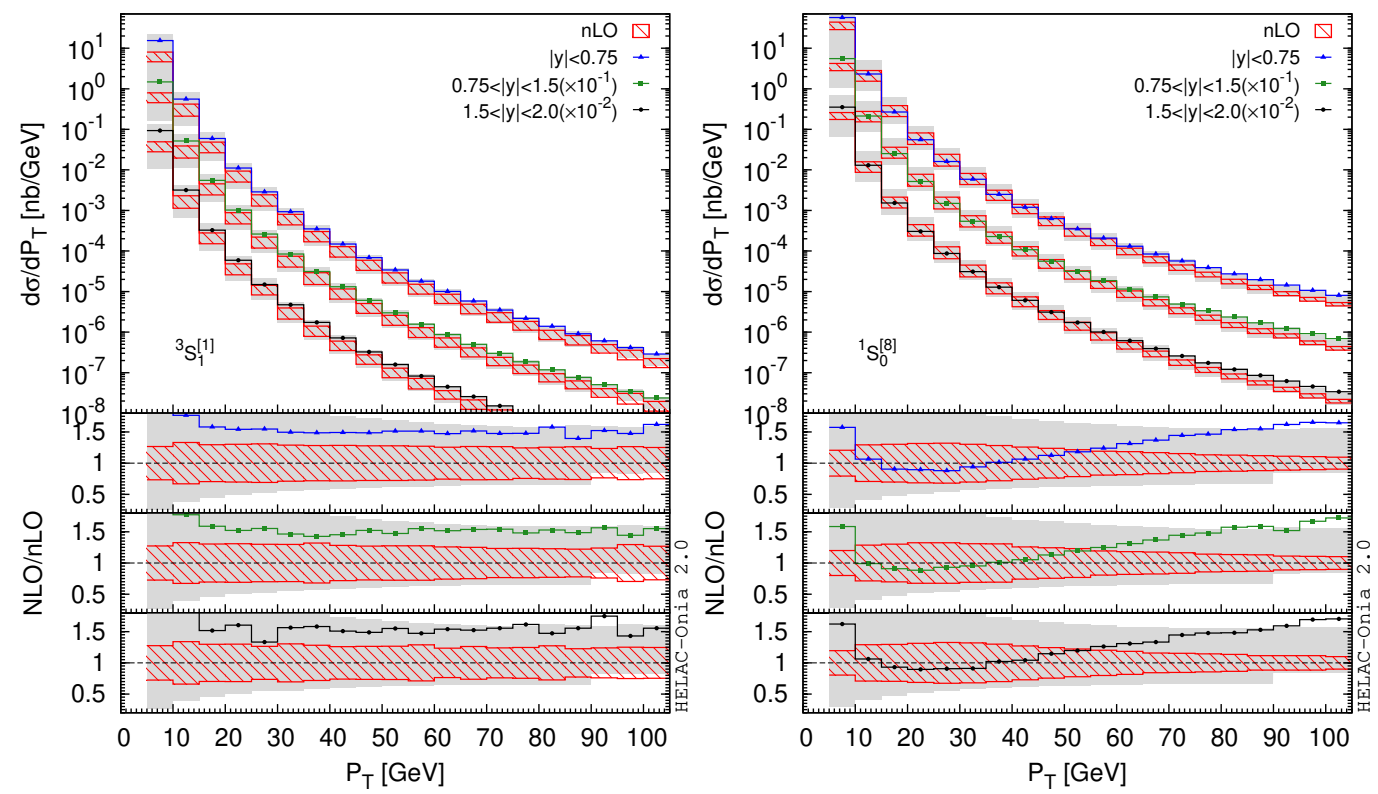

Figure 8. Comparisons of spin-summed differential cross sections for the Fock states ${ }^{3} S_{1}^{[1]},{ }^{1} S_{0}^{[8]}$ between our nLO calculations and the complete NLO calculations.
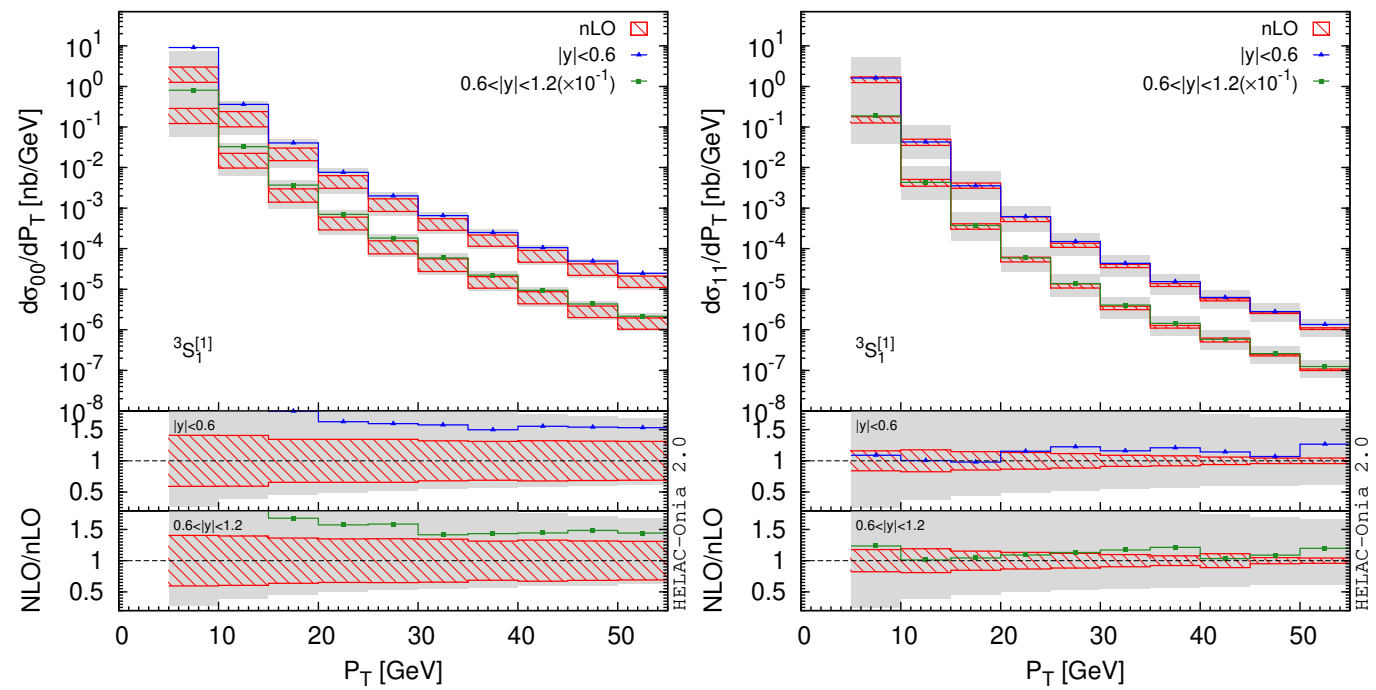

Figure 9. Comparisons of spin-dependent differential cross sections for the Fock state ${ }^{3} S_{1}^{[1]}$ between our nLO calculations and the complete NLO calculations.

${ }^{3} S_{1}^{[1]}$ from NLO to NNLO might be possible in $J / \psi$ production, though the NLO calculation shows that the ${ }^{3} S_{1}^{[1]}$ contribution to $J / \psi$ hadroproduction seems to be negligible compared to the color-octet contributions. If it is true, the extractions of color-octet NRQCD LDMEs solely based on NLO calculations will be questionable. This is one of the reasons why the importance of color-octet contributions in $J / \psi$ hadroproduction is still under debate. Although the accomplishment of NNLO calculations for ${ }^{3} S_{1}^{[1]}$ is still beyond state-of-the art, it was indeed suggested in ref. [3] that the partial calculation shows a giant $\mathrm{K}$ factor $\frac{d \sigma^{\mathrm{NNLO}}}{d \sigma^{\mathrm{NLO}}}$. 


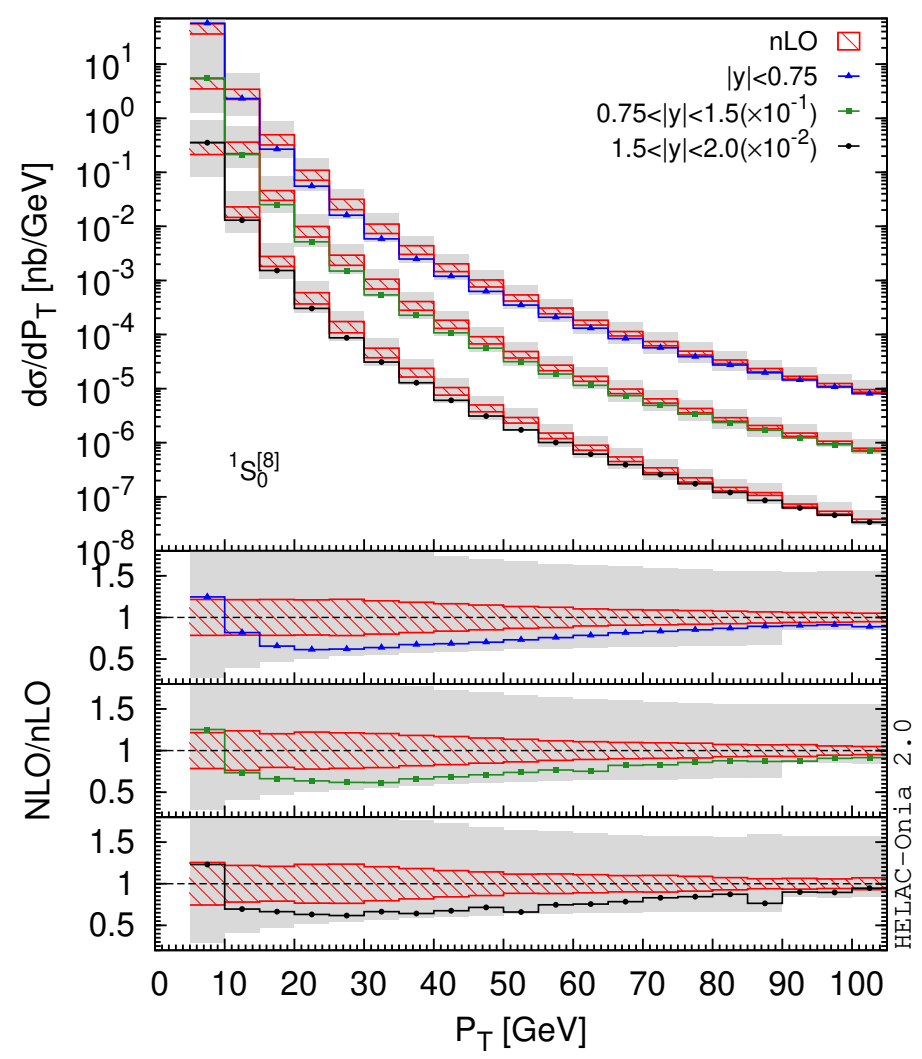

Figure 10. Comparisons of the differential cross sections for ${ }^{1} S_{0}^{[8]}$ between our nLO calculations with $z_{\mathrm{cut}, \mathrm{s}}=\frac{10^{-2}}{m}$ and the complete NLO calculations.

Later on, it was pointed out in ref. [11] that the giant $K$ factor observed in ref. [3] is in fact due to the logarithmic enhancement induced by the infrared cutoff. Such a logarithm is expected to be absent in a full NNLO calculation because of the infrared safety.

We have the opportunity to clarify the situation with our infrared-safe STOP cut method. With the same setup done in section 4.2, we have performed the calculations for ${ }^{3} S_{1}^{[1]}$ plus three light-flavored jets production at $\mathcal{O}\left(\alpha_{s}^{5}\right)$ at the $13 \mathrm{TeV}$. The spin-summed $P_{T}$ differential distributions are shown in figure 12, where we have used "nnLO" and "nNLO" for the $\mathcal{O}\left(\alpha_{s}^{n}\right), n \leq 4$ parts being $\mathrm{nLO}$ and NLO cross sections respectively. In other words, we have used $d \sigma^{\mathrm{nnLO}} \equiv d \sigma^{\mathrm{nLO}}+d \sigma^{\mathcal{R}_{\mathrm{STOP}}^{2}}$ and $d \sigma^{\mathrm{nNLO}} \equiv d \sigma^{\mathrm{NLO}}+d \sigma^{\mathcal{R}_{\mathrm{STOP}}^{2}}$. In the nNLO results, no theoretical uncertainties are taking into account from the NLO piece $d \sigma^{\mathrm{NLO}}$. In contrast to the finding made in ref. [3], we do not observe any giant $\mathrm{K}$ factor up to $P_{T} \simeq 100 \mathrm{GeV}$. In fact, the $P_{T}$ spectra of nnLO and nNLO are not harder than NLO ones. Such an observation can be explained if the coefficient of the LP $P_{T}$ part arising from the single-gluon fragmentation is much smaller than the coefficient of the NLP $P_{T}$ part and/or if the average momentum fraction of ${ }^{3} S_{1}^{[1]}$ taking from the original gluon is significantly smaller than 1 . The calculation based on the gluon fragmentation function shows a similar behaviour, and the normalization of ${ }^{3} S_{1}^{[1]}$ is significantly smaller than the color-octet contributions [43]. In our calculation, the $\mathrm{K}$ factor $\frac{d \sigma^{\mathrm{nnLO}}}{d \sigma^{\mathrm{NLO}}}$ is ranging from 1 to 


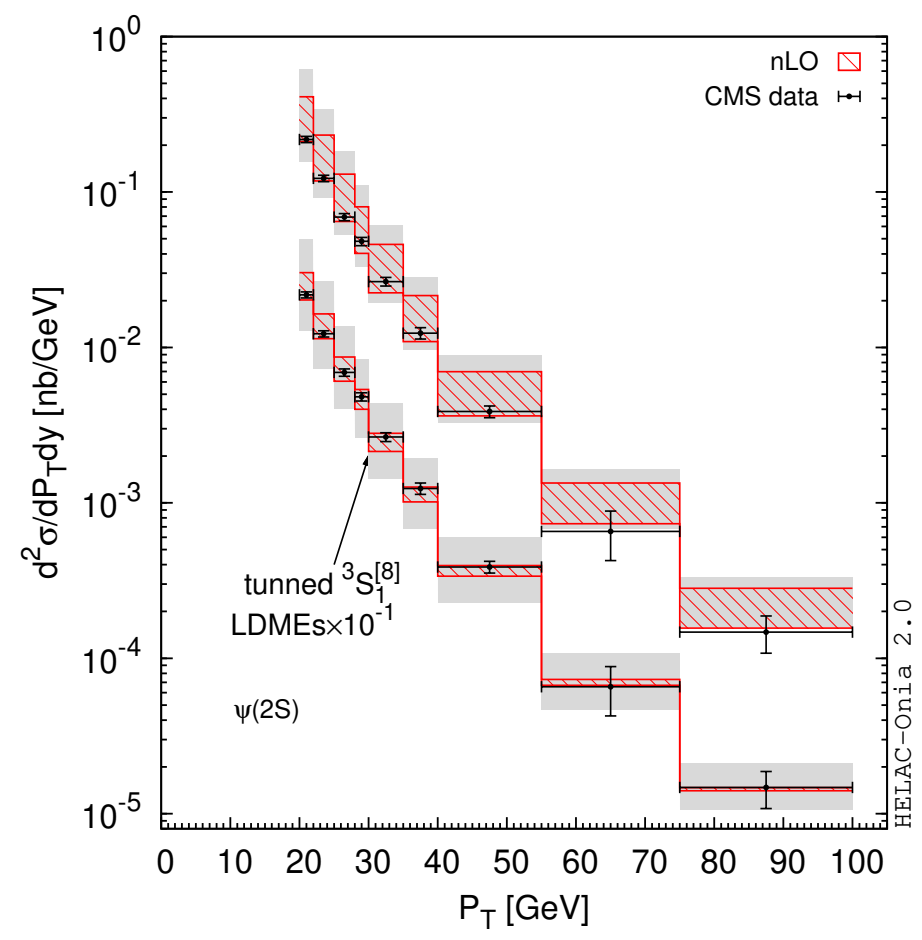

Figure 11. Comparisons of the $\mathrm{nLO} \psi(2 S)$ differential cross sections $\frac{d^{2} \sigma}{d P_{T} d y}$ in $|y|<0.6$ with the CMS measurement [41].

3 depending on the infrared cutoff choices. A similar conclusion can be drawn for the spindependent differential distributions from figure 13. We believe a complete NLO calculations of ${ }^{3} S_{1}^{[1]}$ plus two jets will help to reduce the remaining large infrared cutoff as well as the renormalization/factorization scale dependence.

\subsection{Reassessing the charm fragmentation}

So far, we have only considered the light-flavoured jet(s) accompanying with the quarkonium, which is usually thought to be dominant because the gluons are more often produced than the heavy quarks at high-energy hadron colliders. However, since the LP $P_{T}$ contribution from the charm quark fragmentation appears at $\mathcal{O}\left(\alpha_{s}^{4}\right)$, one should not overlook the associated production processes of a quarkonium plus a heavy quark pair. They were first studied in ref. [44] for ${ }^{3} S_{1}^{[1]}$, in ref. [45] for ${ }^{3} S_{1}^{[8]},{ }^{1} S_{0}^{[8]},{ }^{3} P_{J}^{[8]}$ and in ref. [46] for ${ }^{3} P_{0}^{[1]},{ }^{3} P_{1}^{[1]},{ }^{3} P_{2}^{[1]}$. To the best of our knowledge, the existing calculations only focus on the spin-summed differential cross sections, while we will also present the spin-dependent results in this section. In fact, one has to examine the relevance of these contributions if large cancellations between various Fock states happen.

In figure 14 and figure 15, we compared the tree-level ${ }^{3} S_{1}^{[1]}+c \bar{c}$ (tagged as " $c \vec{c}$ ") production with the nnLO calculations of ${ }^{3} S_{1}^{[1]}$ plus light-flavoured partons. The ${ }^{3} S_{1}^{[1]}+c \bar{c}$ contribution has a harder $P_{T}$ spectrum than the nnLO contribution. The former one exceeds the latter one above $P_{T} \simeq 55 \mathrm{GeV}$ in the spin-summed case, while such a kind of crossover happens earlier for the spin transverse component $\frac{d \sigma_{11}}{d P_{T}}$ around $P_{T} \simeq 20 \mathrm{GeV}$. 

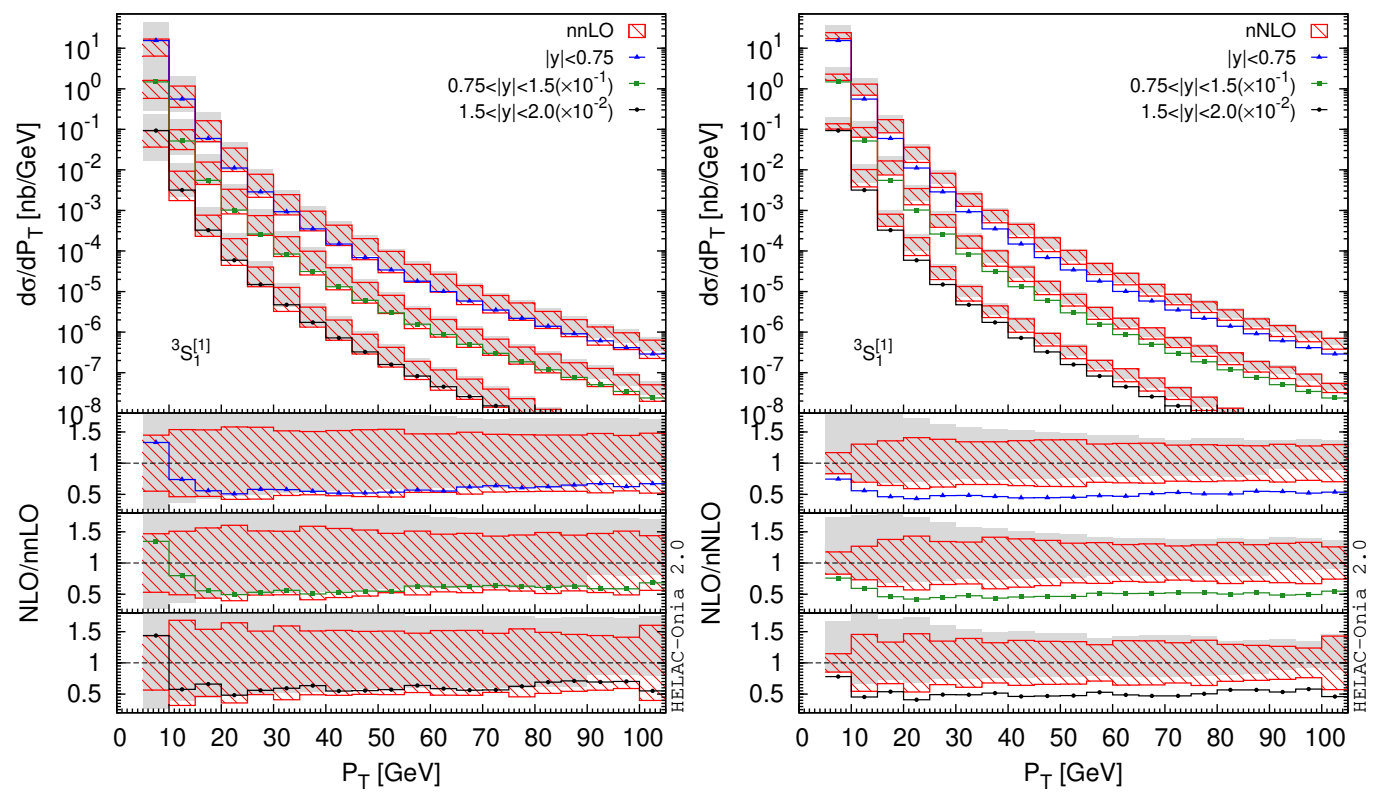

Figure 12. Comparisons of spin-summed differential cross sections for the Fock state ${ }^{3} S_{1}^{[1]}$ between $\mathrm{nnLO}$ (left), nNLO (right) calculations and the complete NLO calculations.
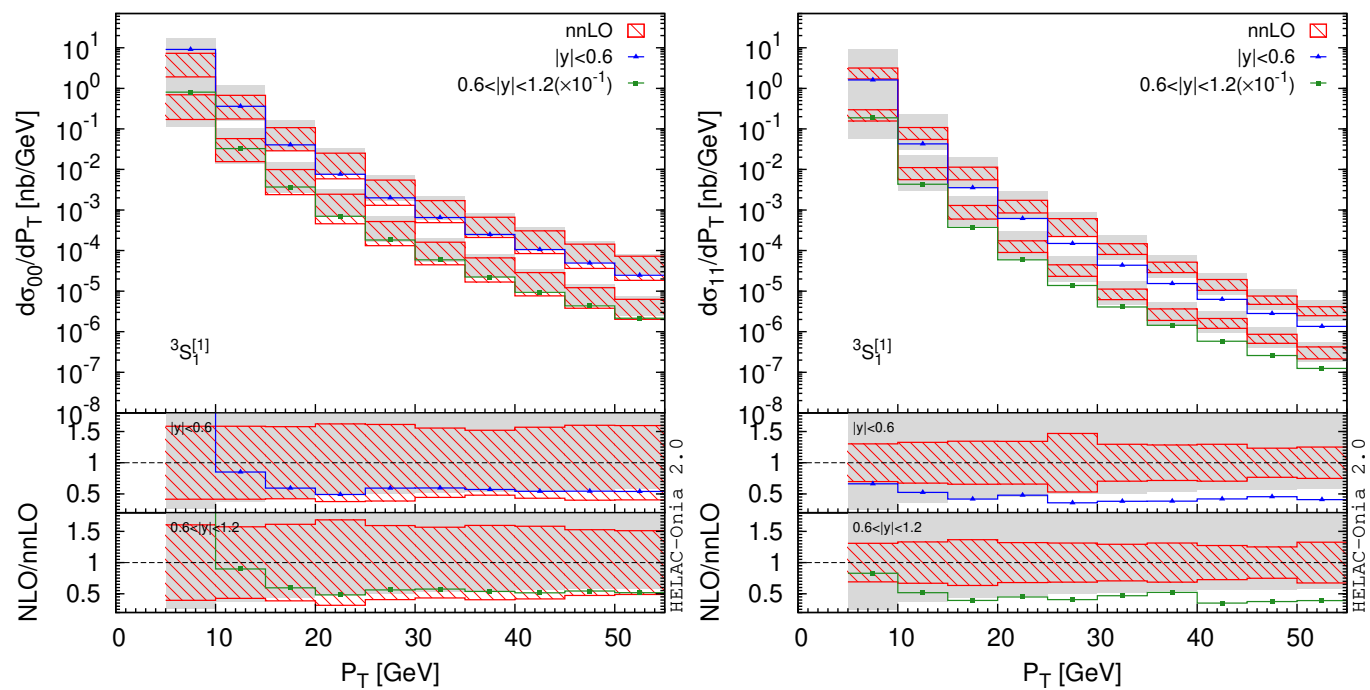

Figure 13. Comparisons of spin-dependent differential cross sections for the Fock state ${ }^{3} S_{1}^{[1]}$ between nnLO calculations and the complete NLO calculations.

On the other hand, the charm quark associated contributions are orders of magnitude smaller than the light-flavoured jet contributions for ${ }^{3} S_{1}^{[8]}$ productions as clearly shown in figure 16 and figure 17 for the spin-summed and spin-dependent distributions. The similar conclusion can be drawn for the other Fock states ${ }^{1} S_{0}^{[8]},{ }^{3} P_{J}^{[8]},{ }^{3} P_{0}^{[1]},{ }^{3} P_{1}^{[1]},{ }^{3} P_{2}^{[1]}$ as shown in figures 24, 25, 26 in the appendix B. 


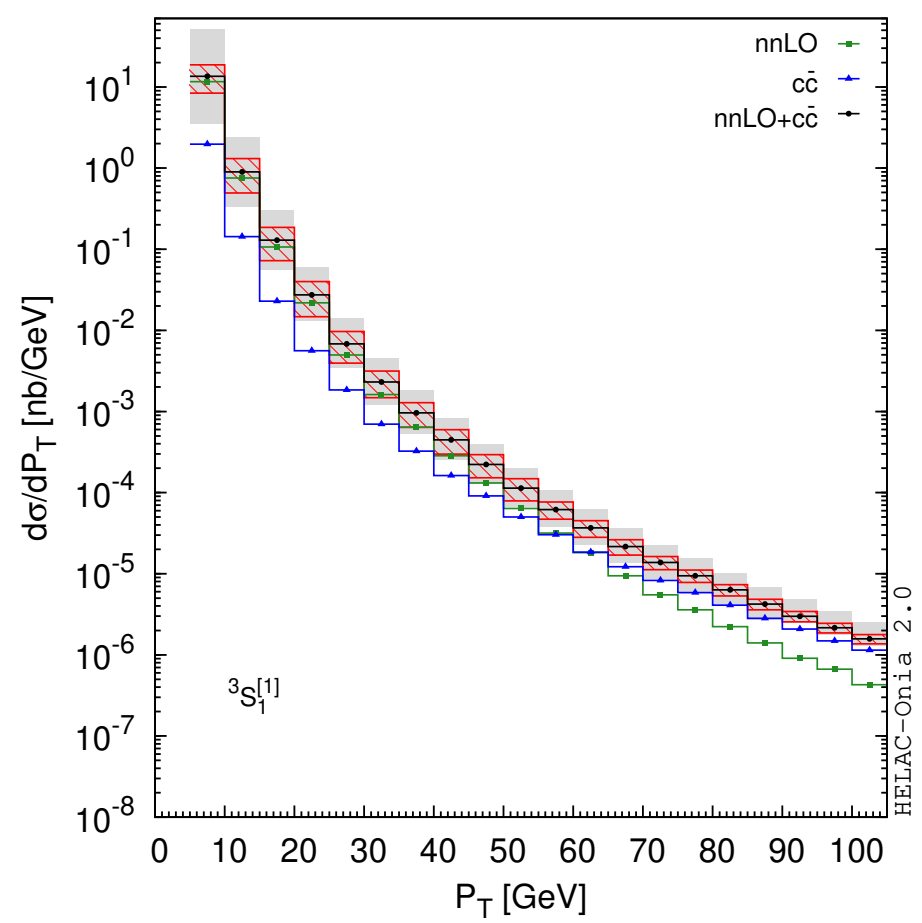

Figure 14. Comparisons of spin-summed differential cross section $\frac{d \sigma}{d P_{T}}$ for the Fock state ${ }^{3} S_{1}^{[1]}$ between our nnLO calculations and the LO charmonium plus charm quark pair calculations.
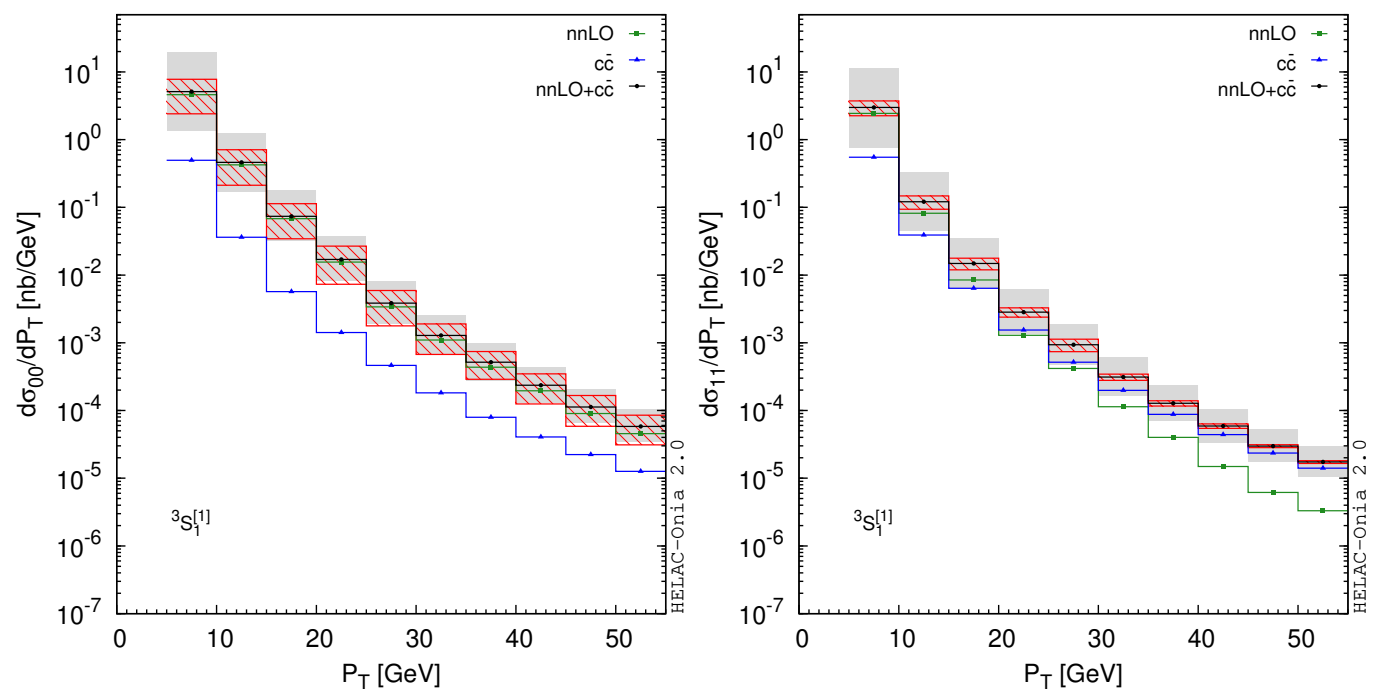

Figure 15. Comparisons of spin-dependent differential cross section $\frac{d \sigma}{d P_{T}}$ for the Fock state ${ }^{3} S_{1}^{[1]}$ between our nnLO calculations and the LO charmonium plus charm quark pair calculations. 


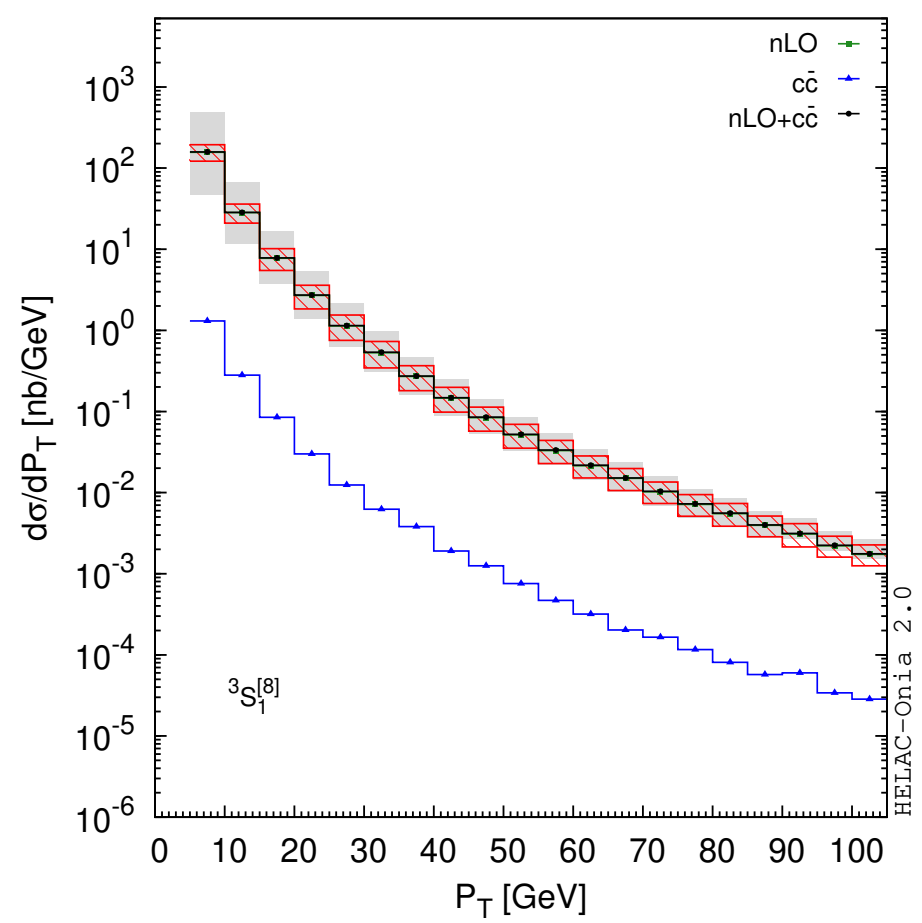

Figure 16. Comparisons of spin-summed differential cross sections $\frac{d \sigma}{d P_{T}}$ for the Fock state ${ }^{3} S_{1}^{[8]}$ between our nLO calculations and the LO charmonium plus charm quark pair calculations.
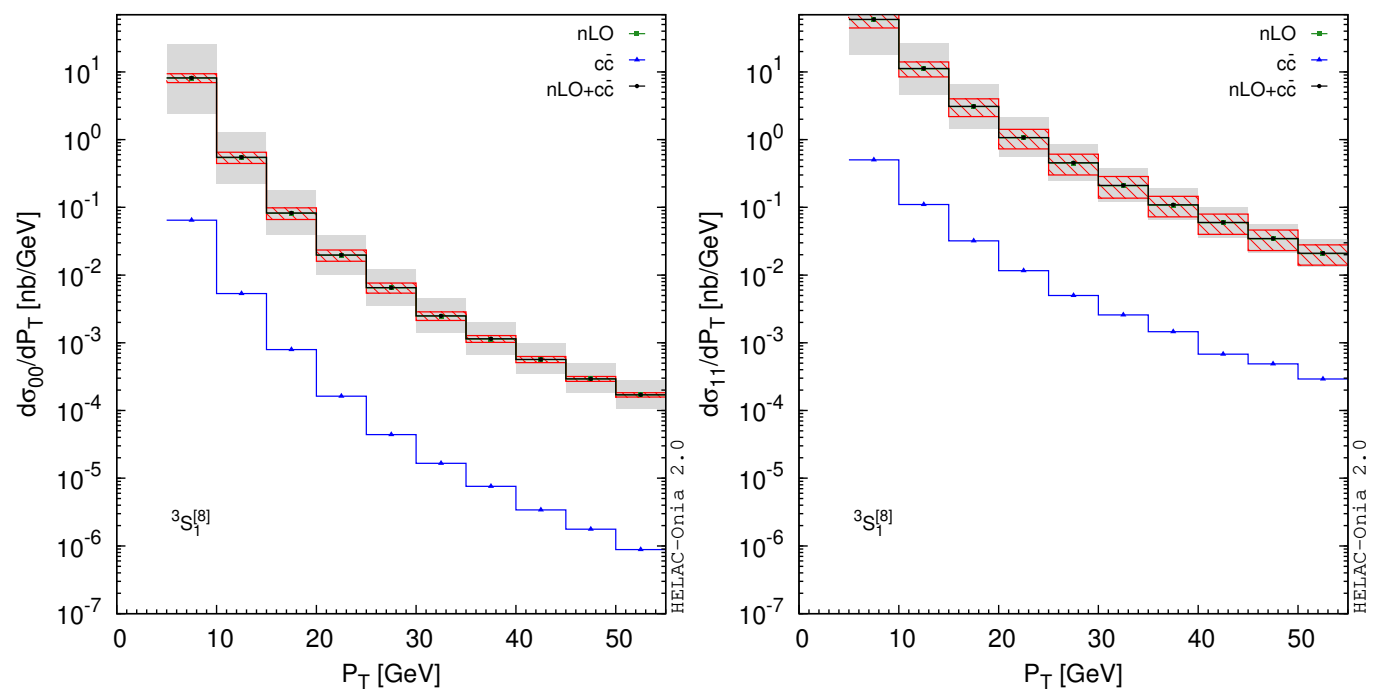

Figure 17. Comparisons of spin-dependent differential cross sections $\frac{d \sigma}{d P_{T}}$ for the Fock state ${ }^{3} S_{1}^{[8]}$ between our $\mathrm{nLO}$ calculations and the LO charmonium plus charm quark pair calculations. 


\section{$5 \quad$ Summary and outlooks}

After implementing the remainders of $\mathrm{P}$-wave counterterms in section 2, we have introduced a general infrared-safe method to estimate the giant $\mathrm{K}$ factors in quarkonium production in high $P_{T}$ region. As a proof of concept, we have validated our approach with the existing complete NLO QCD calculations of the Fock states ${ }^{3} S_{1}^{[1,8]},{ }^{1} S_{0}^{[8]},{ }^{3} P_{J}^{[1,8]}$ in both spin-summed and spin-dependent cases. They are relevant for $J / \psi$ and $\chi_{c J}$ hadroproduction up to $\mathcal{O}\left(v^{7}\right)$. Our approach only requires the tree-level amplitudes provided by HELAC-ONIA. To the best of our knowledge, it is the first time to be able to reproduce the complete NLO spin-dependent results with tree-level amplitudes only. These spin-dependent results can be used to predict the polarization observables. We are also firstly able to obtain the spin-summed NLO results for ${ }^{3} P_{J}^{[1]}$ without performing complete NLO calculations. With our new approach, we have estimated the partial NNLO contributions at $\mathcal{O}\left(\alpha_{s}^{5}\right)$ for ${ }^{3} S_{1}^{[1]}$ production. It is believed to be at LP in $P_{T}$ scaling starting at this order, and is the last missing piece for the heavy quarkonium $P_{T}$ spectrum up to $\mathcal{O}\left(v^{7}\right)$. In contrast to the $\mathrm{NNLO}^{\star}$ calculations based on the simple invariant-mass cuts [3], we do not observe the similar orders of magnitude enhancement compared to the NLO calculations, while an enhancement factor of 1 to 3 is still possible up to $P_{T} \simeq 100 \mathrm{GeV}$ depending on the infrared cutoff choices. We believe the complete NLO calculations of ${ }^{3} S_{1}^{[1]}$ plus 2 jets will reduce this uncertainty. Finally, we have also calculated the charmonium plus a charm quark pair production, where the spin-dependent differential cross sections presented here are new. Their contributions to the inclusive $P_{T}$ distributions of charmonium are only relevant in the ${ }^{3} S_{1}^{[1]}$ channel.

Our approach stabilizes the QCD corrections in the heavy quarkonium production rate calculations at high $P_{T}$. It is quite appealing not only because it provides a fast way to perform the phenomenology studies of inclusive quarkonium production but also it can be used to improve the predictions in the associated quarkonium production processes. Together with the controlled perturbative SDCs, it is feasible to study various nonperturbative effects in the heavy quarkonium hadroproduction in an acceptable amount of computation time. Last but not least, with a similar method, we believe that we are able to promote the accuracy of both LP and NLP pieces to NLO level simultaneously with the full one-loop calculations.

\section{Acknowledgments}

I thank Jean-Philippe Lansberg for useful discussions. This work is supported by the ILP Labex (ANR-11-IDEX-0004-02, ANR-10-LABX-63). The computations in this paper were performed with the help of the computing facilities at IPN Orsay.

\section{A Calculations with HELAC-ONIA}

In this section, we will give an instruction on how to use HELAC-ONIA to perform nLO and nnLO calculations. The implementations are available from version 2.3.6 and onwards, 
which can be downloaded from http://hshao.web.cern.ch/hshao/helaconia.html. An example of a FORTRAN analysis file plot_pp_psinjets_spin2.f90 is given in the subdirectory analysis/user in order to get the differential distributions in this paper. The common calculation setup is given by the following entries in user.inp:

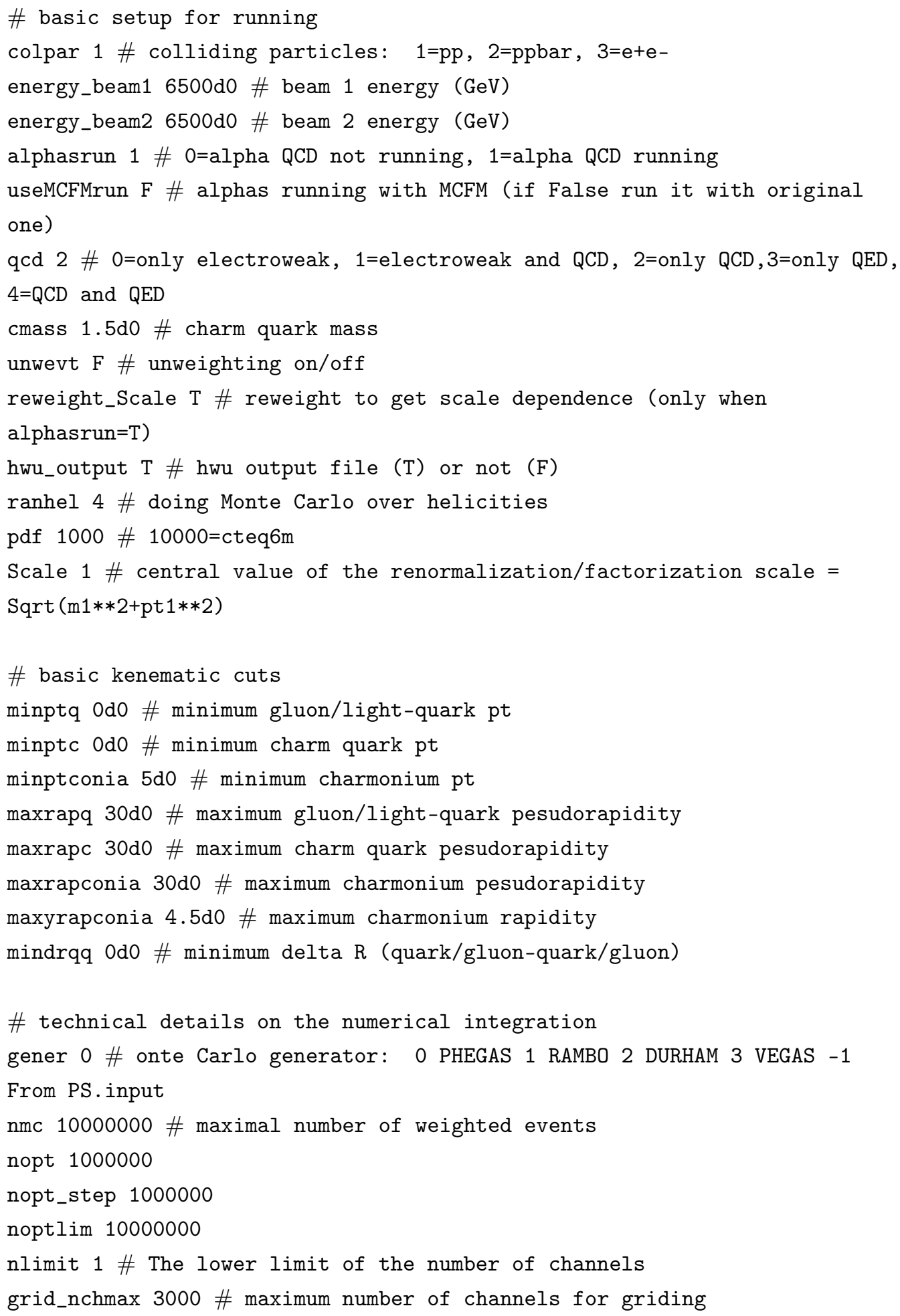




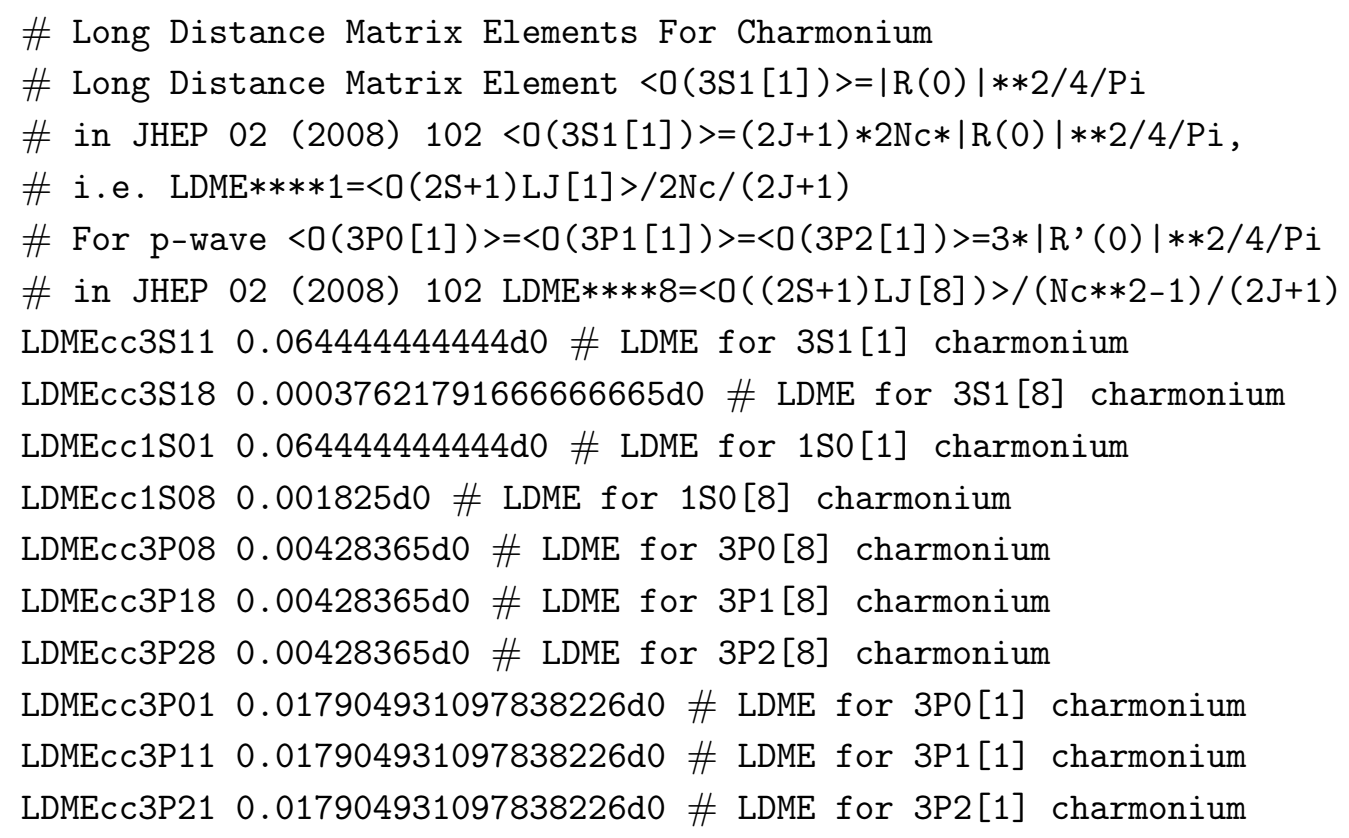

\section{A.1 Born and counterterms}

The Born $d \sigma^{\mathcal{B}}$ at $\mathcal{O}\left(\alpha_{s}^{3}\right)$ for S-wave Fock states can be achieved via the following commands:

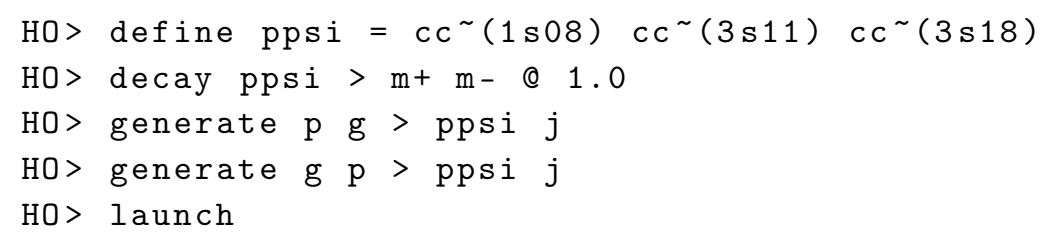

where we have always excluded the quark-quark initial states due to their very small parton luminosity from PDFs. No extra kinematical cuts are needed for the Born-like events.

The color-octet $\mathrm{P}$-wave Fock states ${ }^{3} P_{J}^{[8]}$ can be grouped together as we will always use the relation from heavy-quark spin symmetry $\left\langle\mathcal{O}\left({ }^{3}{ }^{[8]}\right)\right\rangle=(2 J+1)\left\langle\mathcal{O}\left({ }^{3} P_{0}^{[8]}\right)\right\rangle$. The contributions from the Born and the remainders of the counterterms $d \sigma^{\mathcal{B}}+d \sigma^{\mathcal{C}}$ can be included via:

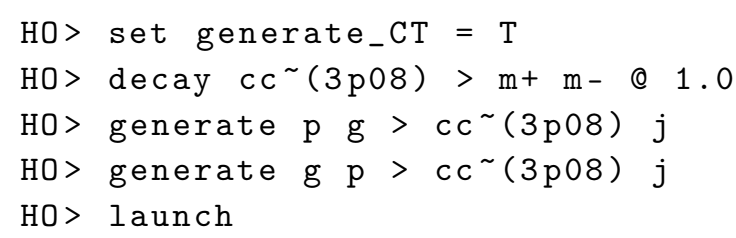

The command sets generate_CT to be $\mathrm{T}$ in order to get the contributions from counterterms $d \sigma^{\mathcal{C}}$.

The color-singlet P-wave Fock states ${ }^{3} P_{J}^{[1]}$ will be calculated separately since they contribute to $\chi_{c J}, J=0,1,2$ respectively. The commands are:

HO> set $\exp 3 p j Q=T$ 


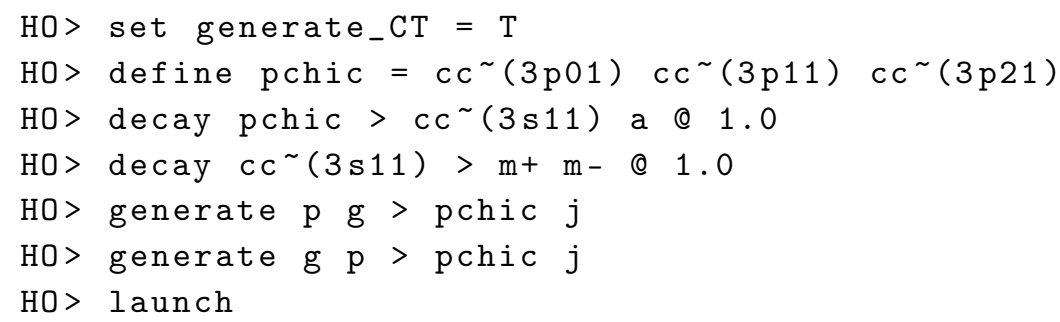

We set $\exp 3 \mathrm{pjQ}=\mathrm{T}$ in order to get ${ }^{3} P_{J}^{[1]}, J=0,1,2$ individually. At the meantime, ${ }^{3} P_{J}^{[1]}$ are cascaded decaying to ${ }^{3} S_{1}^{[1]}+\gamma \rightarrow \mu^{+} \mu^{-}+\gamma$. The counterterms should be taken into account by setting generate_ $\mathrm{CT}=\mathrm{T}$.

\section{A.2 Real terms}

One should apply the STOP cuts to the real terms at $\mathcal{O}\left(\alpha_{s}^{n}\right), n \geq 4$. It requires us to implement the following additional entries in user.inp:

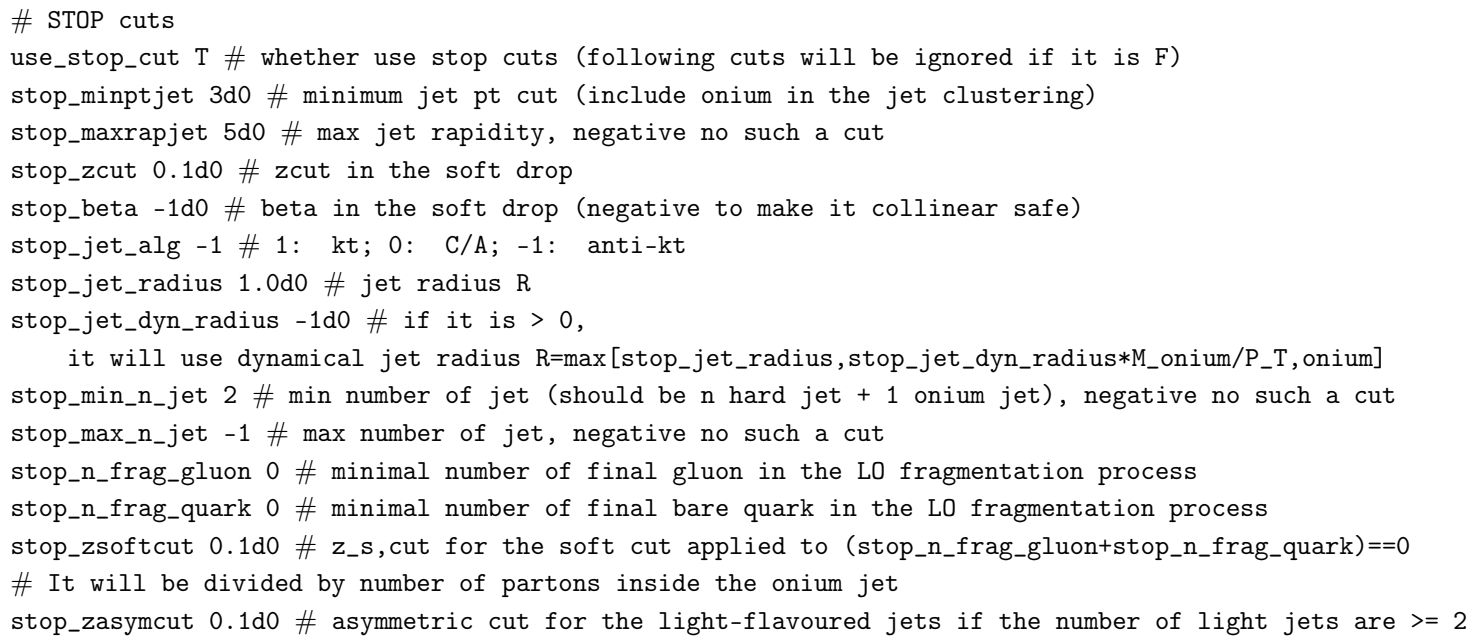

The HELAC-Onia commands to calculate the $\mathcal{O}\left(\alpha_{s}^{4}\right)$ real terms $d \sigma^{\mathcal{R}_{\text {STOP }}}$ are

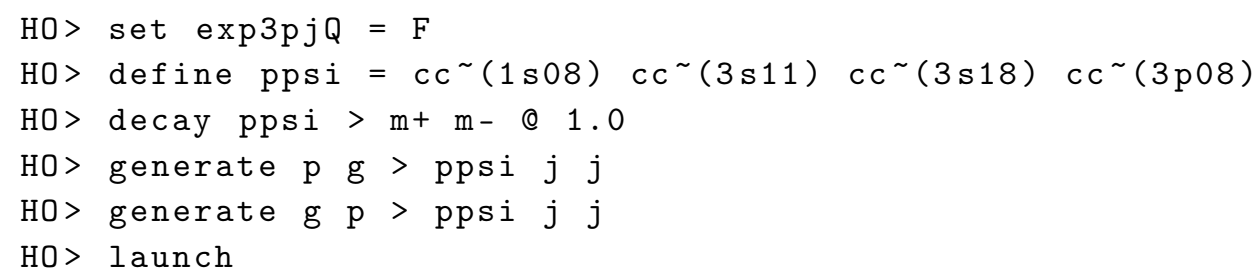

for the S-wave and color-octet P-wave Fock states, while for ${ }^{3} P_{J}^{[1]}$ one should type

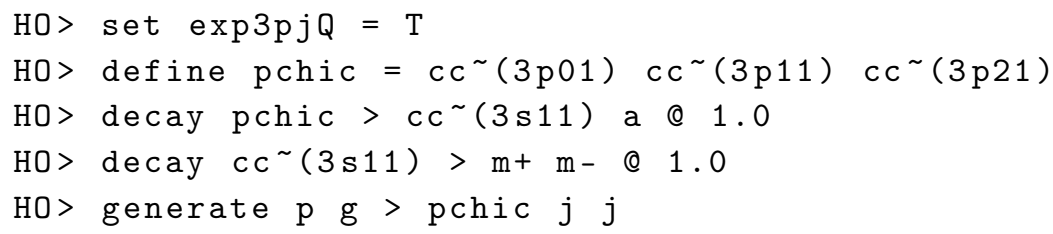


HO $>$ generate g p pchic j j

$\mathrm{HO}>$ launch

With the same STOP cuts, the generation of weighted events at $\mathcal{O}\left(\alpha_{s}^{5}\right) d \sigma^{\mathcal{R}_{\text {STOP }}^{2}}$ for ${ }^{3} S_{1}^{[1]}$ can be achieved by the following commands:

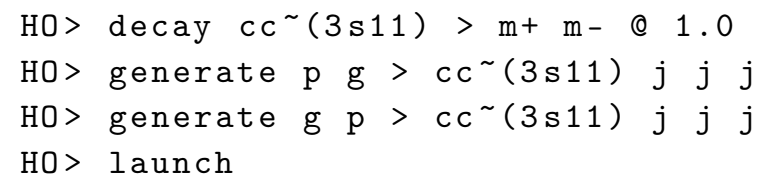

\section{B Supplemental plots}

We provided the supplemental plots in this appendix for the sake of completeness. The comparisons of spin-summed differential cross sections for the 5 Fock states ${ }^{1} S_{0}^{[8]},{ }^{3} P_{J}^{[8]}$, ${ }^{3} P_{0}^{[1]},{ }^{3} P_{1}^{[1]},{ }^{3} P_{2}^{[1]}$ between aNLO and NLO are shown in figure 18 , while the spin-dependent ones for ${ }^{3} P_{J}^{[8]},{ }^{3} P_{1}^{[1]},{ }^{3} P_{2}^{[1]}$ can be found in figure 19 and figure 20 . The $n L O$ versus NLO plots for ${ }^{3} P_{J}^{[8]},{ }^{3} P_{0}^{[1]},{ }^{3} P_{1}^{[1]},{ }^{3} P_{2}^{[1]}$ are available in figure 21 (spin-summed ones) and in figures 22,23 (spin-dependent ones). The contributions from $\mathcal{O}_{n}+c \bar{c}$ with $\mathcal{O}_{n}={ }^{1} S_{0}^{[8]},{ }^{3} P_{J}^{[8]},{ }^{3} P_{0}^{[1]},{ }^{3} P_{1}^{[1]}$, ${ }^{3} P_{2}^{[1]}$ are shown in figures $24,25,26$. 

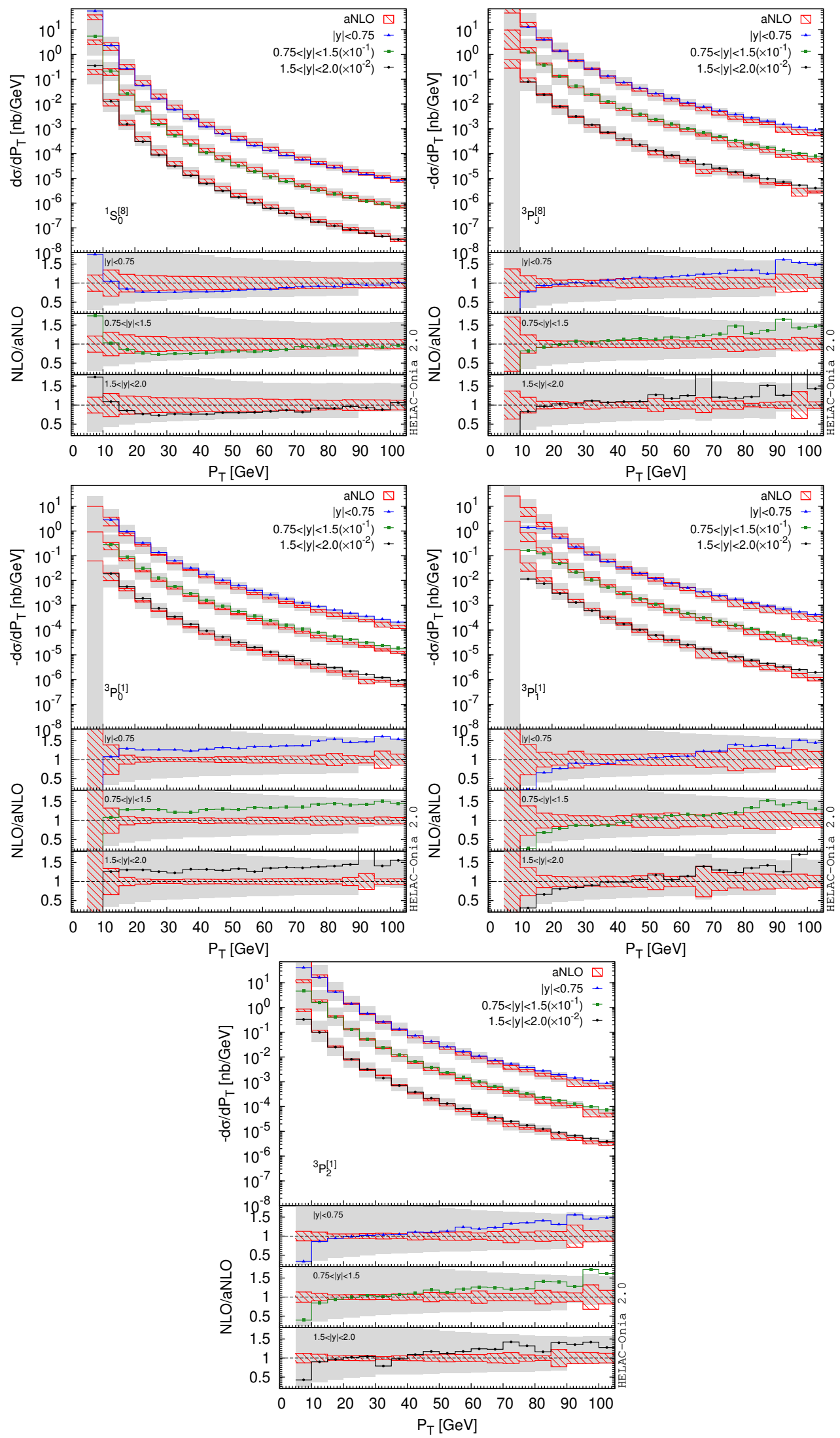

Figure 18. Comparisons of spin-summed differential cross sections for the Fock states ${ }^{1} S_{0}^{[8]},{ }^{3} P_{J}^{[8]}$, ${ }^{3} P_{0}^{[1]},{ }^{3} P_{1}^{[1]},{ }^{3} P_{2}^{[1]}$ between our aNLO calculations and the complete NLO calculations. They are similar to figure 1. 

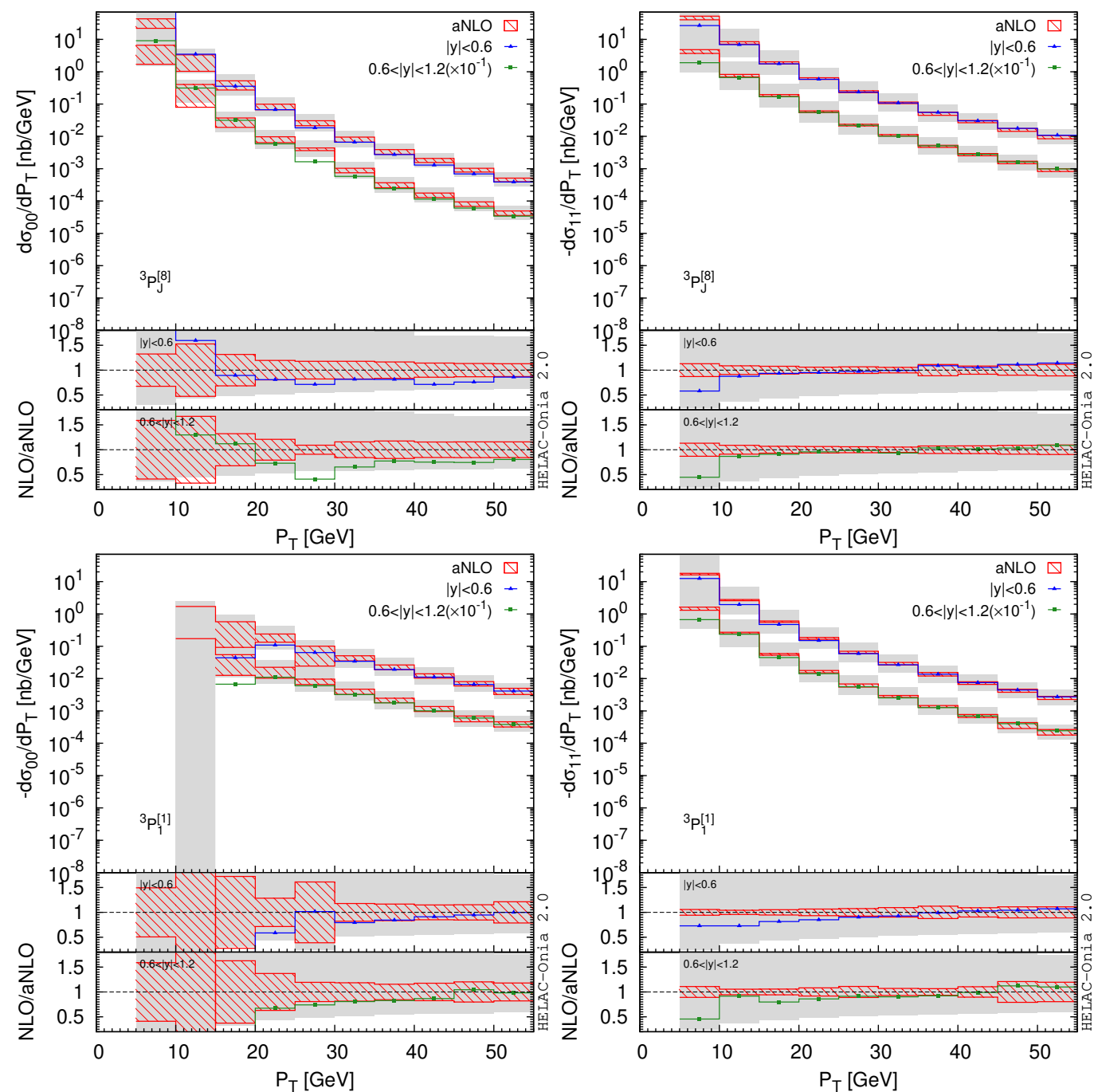

Figure 19. Comparisons of spin-dependent differential cross sections for the 2 Fock states ${ }^{3} P_{J}^{[8]},{ }^{3} P_{1}^{[1]}$ between our aNLO calculations and the complete NLO calculations. They are similar to figure 2 . 

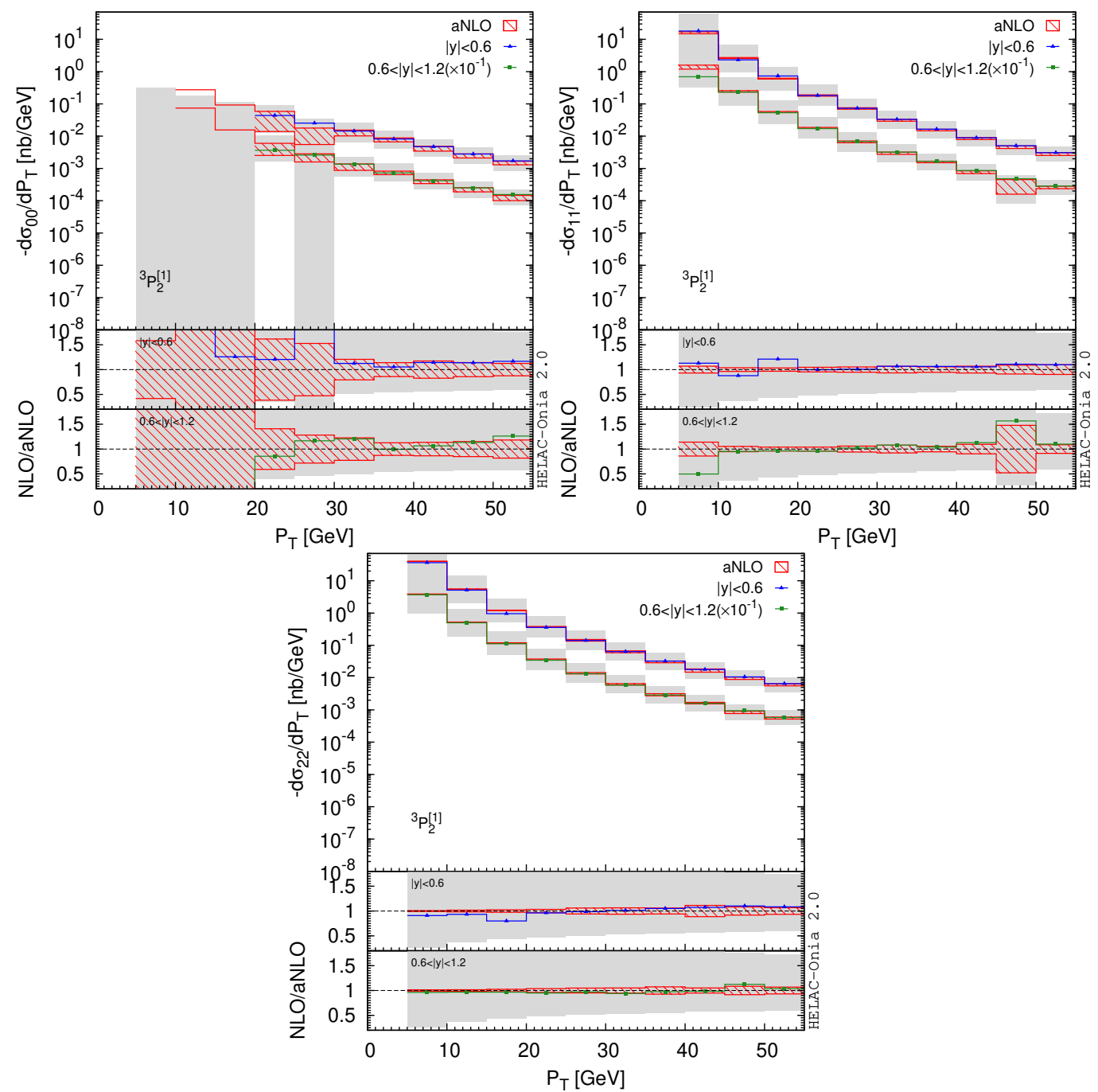

Figure 20. Comparisons of spin-dependent differential cross sections for the Fock state ${ }^{3} P_{2}^{[1]}$ between our aNLO calculations and the complete NLO calculations. They are similar to figure 2 . 

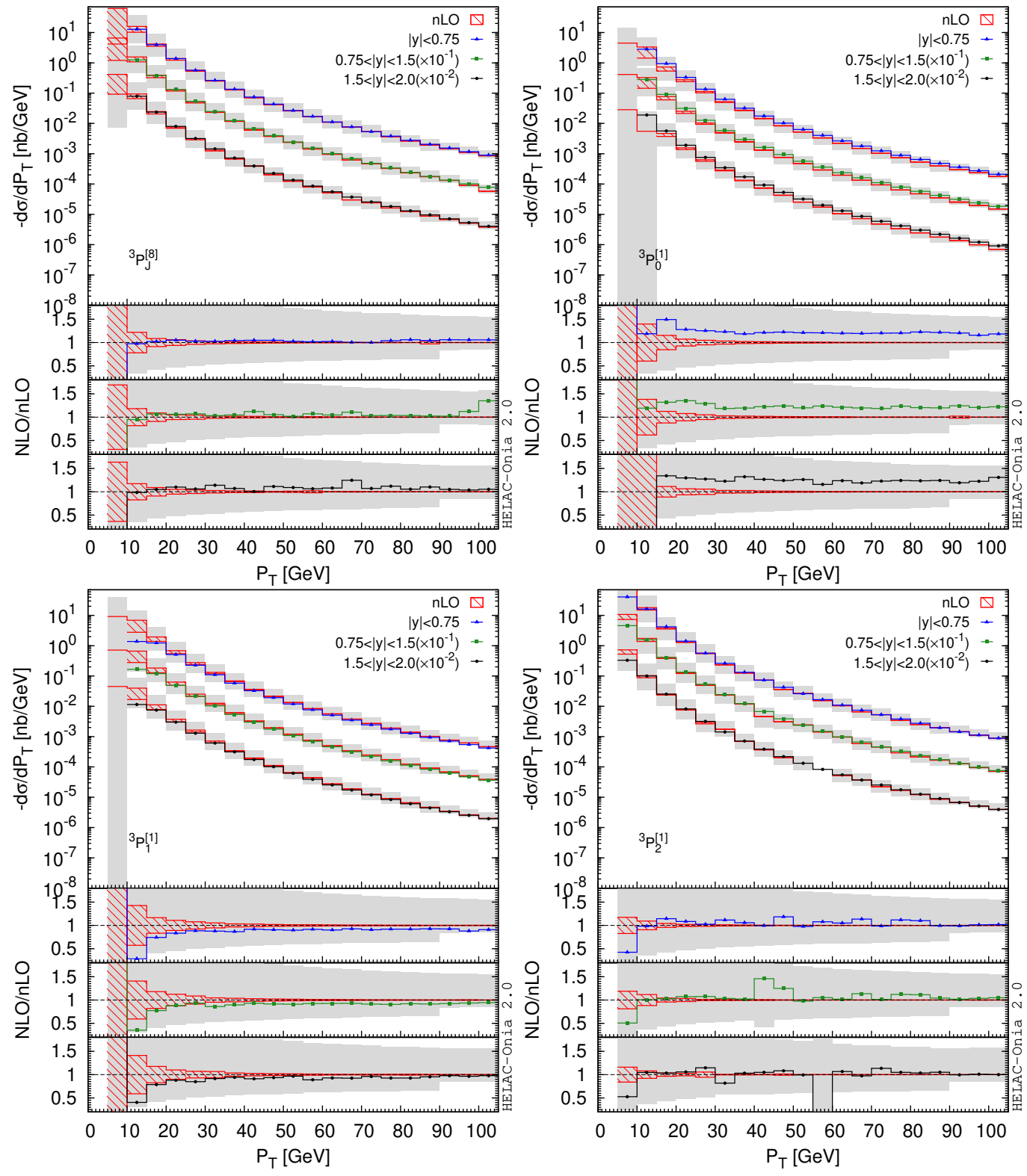

Figure 21. Comparisons of spin-summed differential cross sections for the Fock states ${ }^{3} P_{J}^{[8]},{ }^{3} P_{0}^{[1]}$, ${ }^{3} P_{1}^{[1]},{ }^{3} P_{2}^{[1]}$ between our nLO calculations and the complete NLO calculations. They are similar to figure 8 . 

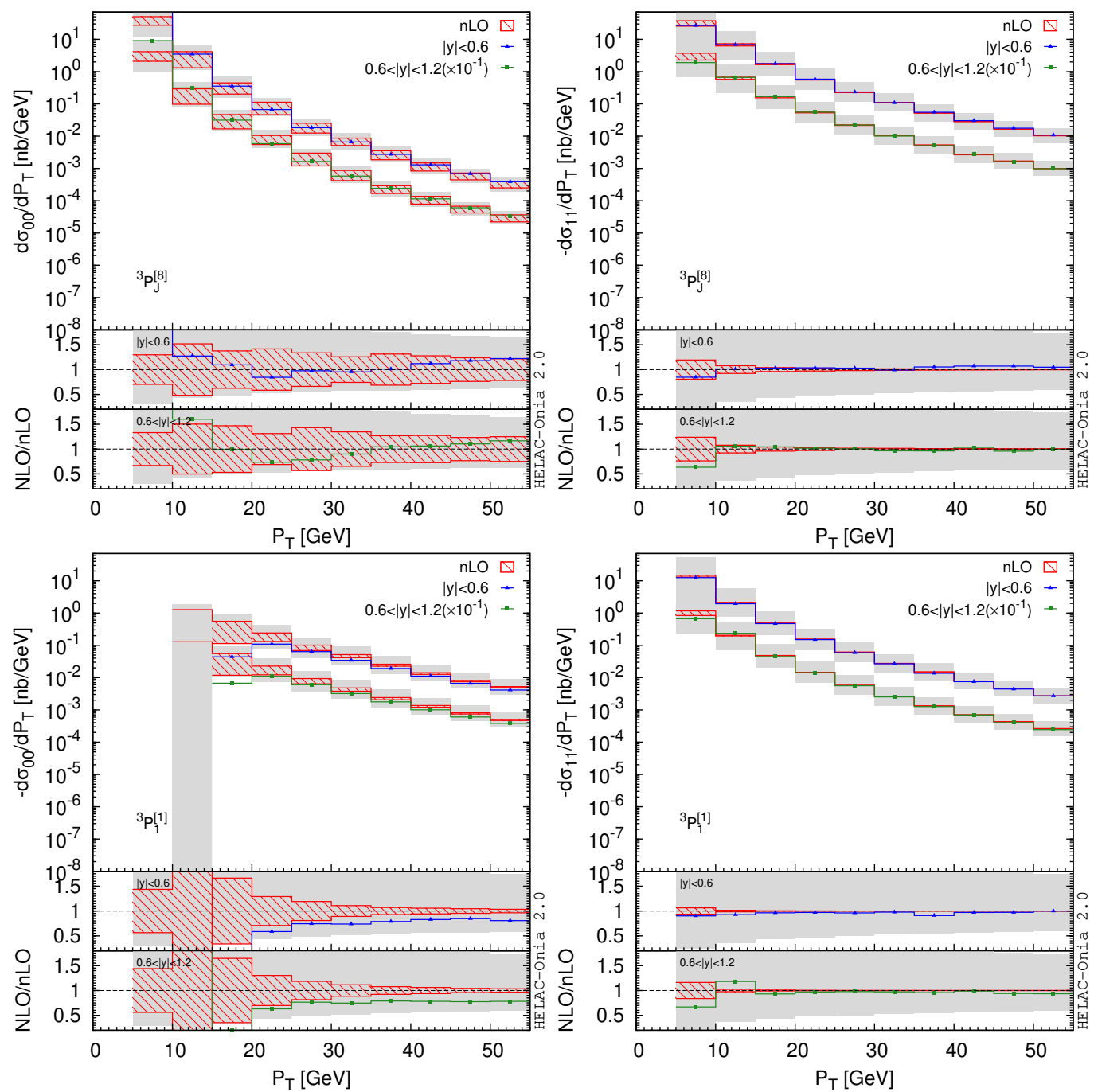

Figure 22. Comparisons of spin-dependent differential cross sections for the 3 Fock states ${ }^{3} P_{J}^{[8]},{ }^{3} P_{1}^{[1]}$ between our nLO calculations and the complete NLO calculations. They are similar to figure 9 . 

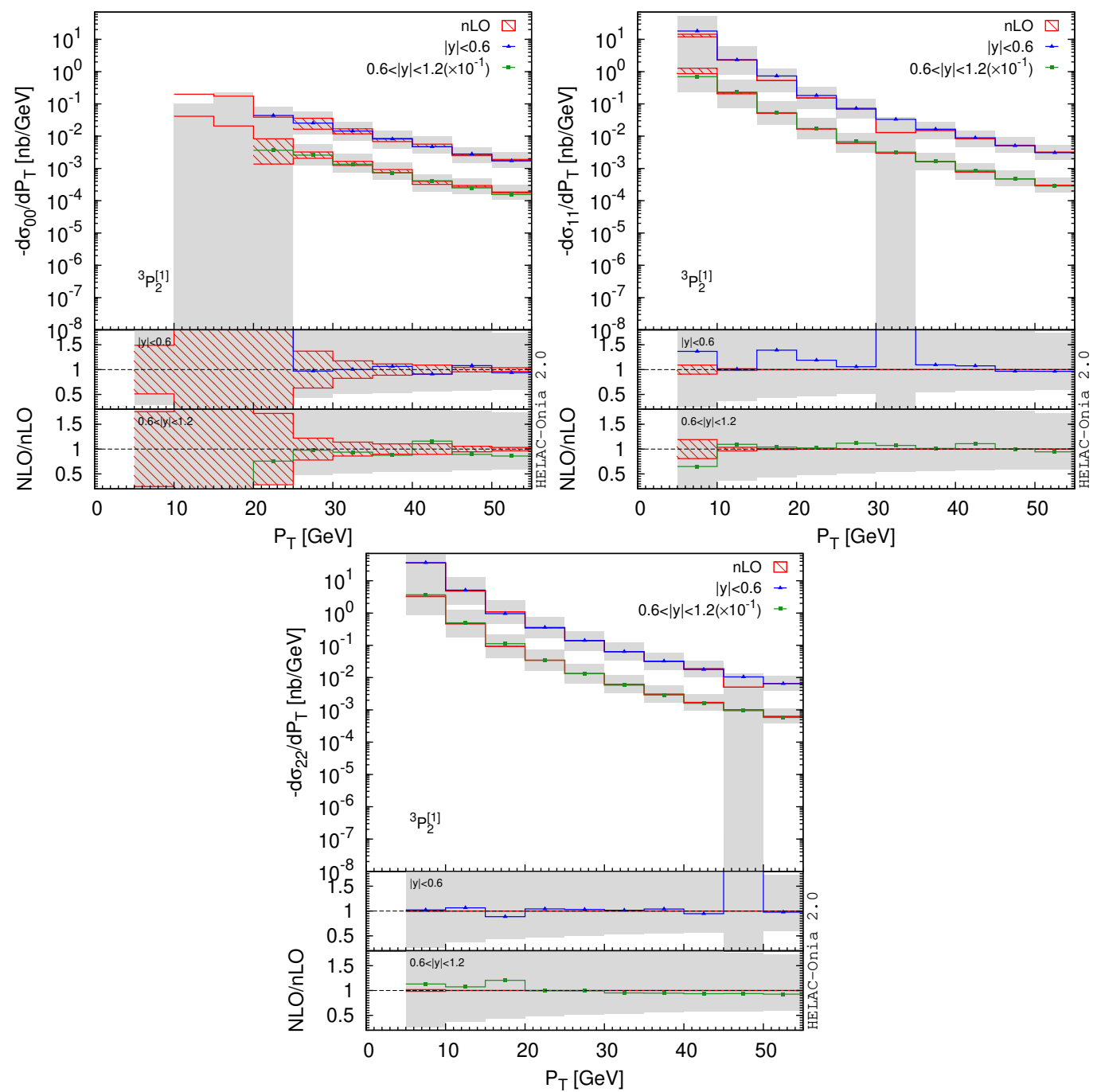

Figure 23. Comparisons of spin-dependent differential cross sections for the Fock state ${ }^{3} P_{2}^{[1]}$ between our nLO calculations and the complete NLO calculations. They are similar to figure 9 . 

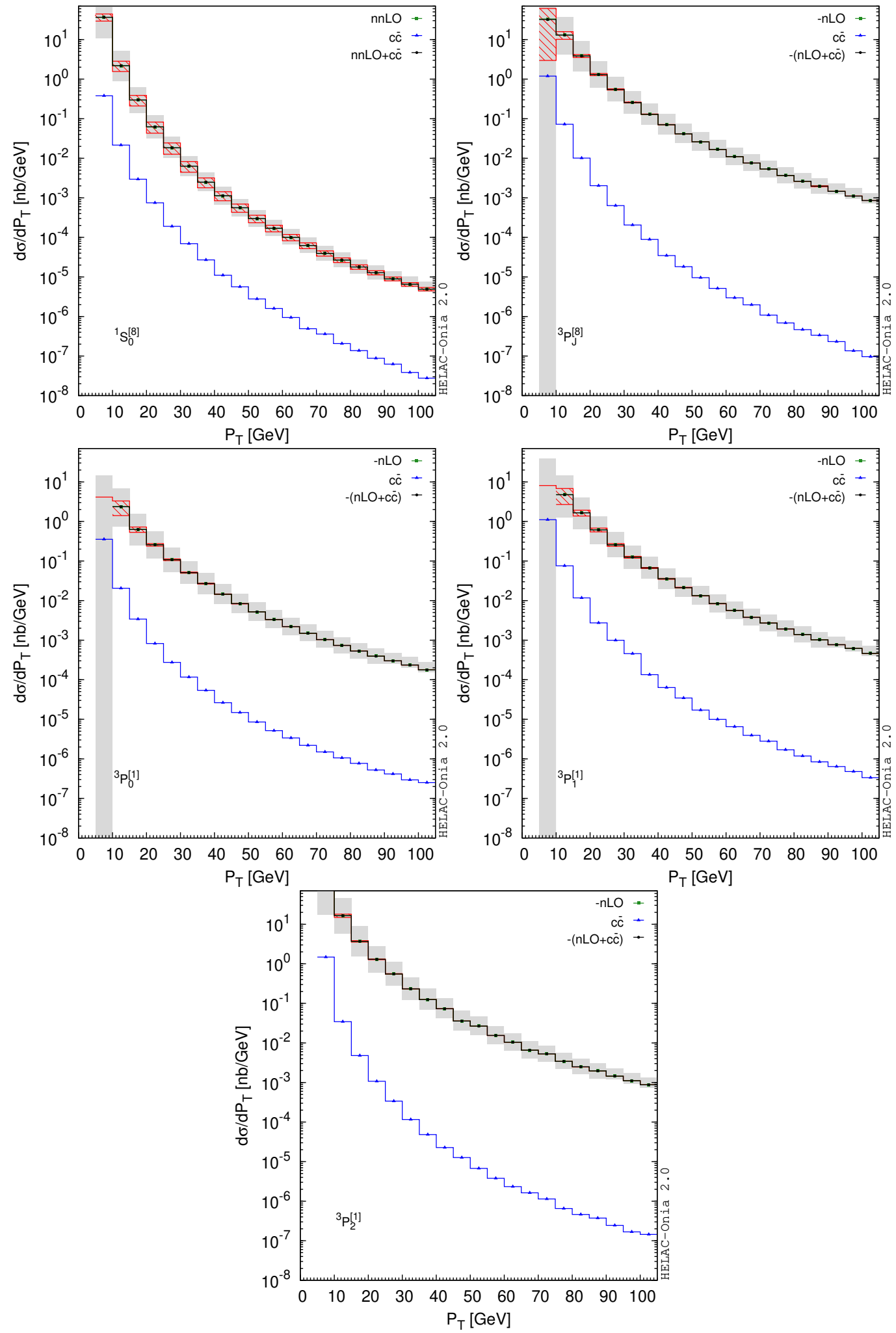

Figure 24. Comparisons of spin-summed differential cross sections $\frac{d \sigma}{d P_{T}}$ for the 5 Fock states ${ }^{1} S_{0}^{[8]},{ }^{3} P_{J}^{[8]},{ }^{3} P_{0}^{[1]},{ }^{3} P_{1}^{[1]},{ }^{3} P_{2}^{[1]}$ between our nLO calculations and the LO charmonium plus charm quark pair calculations. They are similar to figure 16 . 

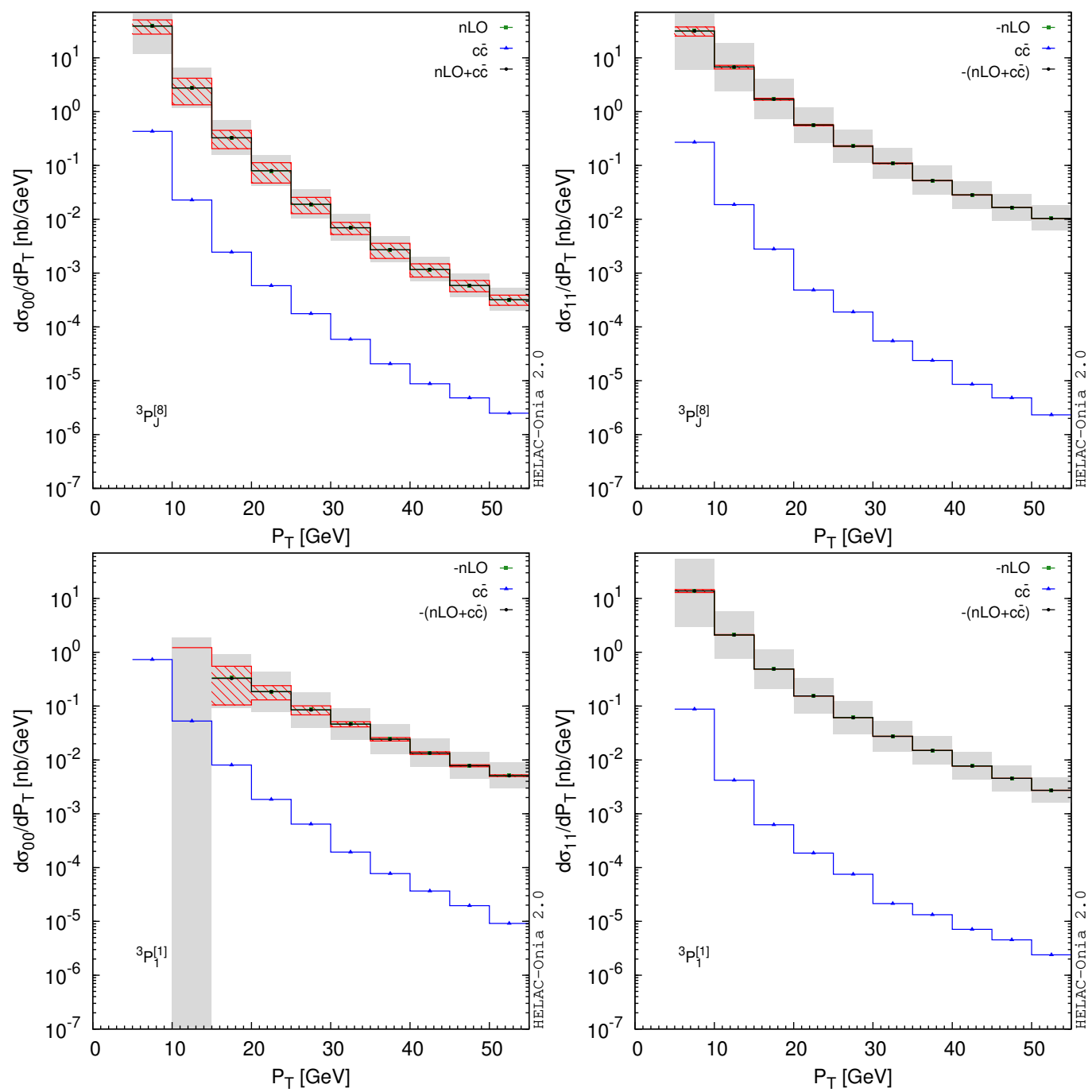

Figure 25. Comparisons of spin-dependent differential cross sections $\frac{d \sigma}{d P_{T}}$ for the Fock states ${ }^{3} P_{J}^{[8]},{ }^{3} P_{1}^{[1]}$ between our nLO calculations and the LO charmonium plus charm quark pair calculations. They are similar to figure 17 . 

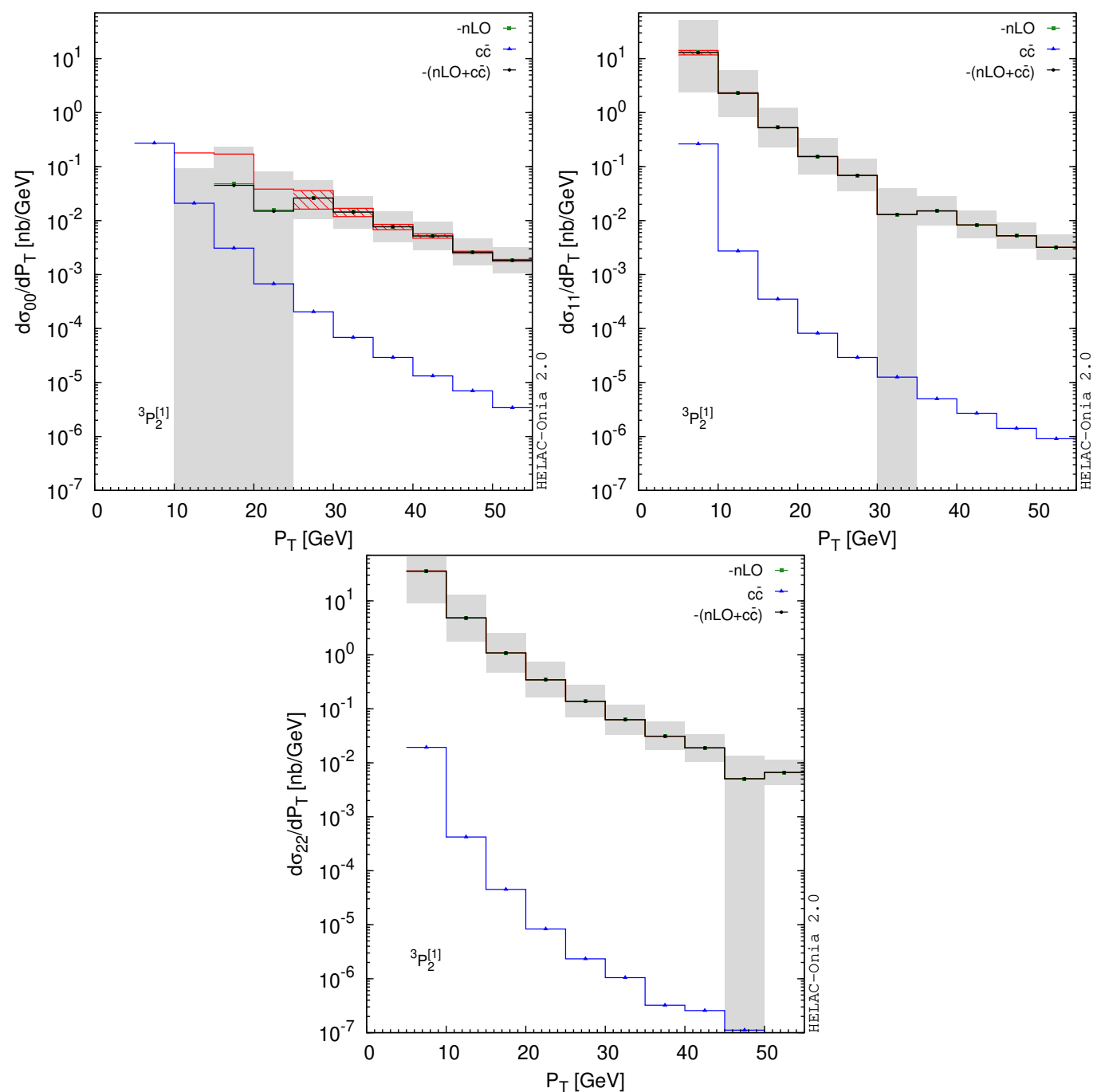

Figure 26. Comparisons of spin-dependent differential cross sections $\frac{d \sigma}{d P_{T}}$ for the Fock state ${ }^{3} P_{2}^{[1]}$ between our nLO calculations and the LO charmonium plus charm quark pair calculations. They are similar to figure 17 . 
Open Access. This article is distributed under the terms of the Creative Commons Attribution License (CC-BY 4.0), which permits any use, distribution and reproduction in any medium, provided the original author(s) and source are credited.

\section{References}

[1] G.T. Bodwin, E. Braaten and G.P. Lepage, Rigorous QCD analysis of inclusive annihilation and production of heavy quarkonium, Phys. Rev. D 51 (1995) 1125 [Erratum ibid. D 55 (1997) 5853] [hep-ph/9407339] [InSPIRE].

[2] J.M. Campbell, F. Maltoni and F. Tramontano, QCD corrections to J/psi and Upsilon production at hadron colliders, Phys. Rev. Lett. 98 (2007) 252002 [hep-ph/0703113] [INSPIRE].

[3] P. Artoisenet, J.M. Campbell, J.P. Lansberg, F. Maltoni and F. Tramontano, $\Upsilon$ Production at Fermilab Tevatron and LHC Energies, Phys. Rev. Lett. 101 (2008) 152001 [arXiv: 0806.3282] [INSPIRE].

[4] J.-P. Lansberg and H.-S. Shao, Production of $J / \psi+\eta_{c}$ versus $J / \psi+J / \psi$ at the LHC: Importance of Real $\alpha_{s}^{5}$ Corrections, Phys. Rev. Lett. 111 (2013) 122001 [arXiv:1308.0474] [INSPIRE].

[5] L.-P. Sun, H. Han and K.-T. Chao, Impact of $J / \psi$ pair production at the $L H C$ and predictions in nonrelativistic QCD, Phys. Rev. D 94 (2016) 074033 [arXiv: 1404.4042] [INSPIRE].

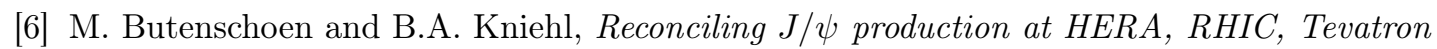
and LHC with NRQCD factorization at next-to-leading order, Phys. Rev. Lett. 106 (2011) 022003 [arXiv: 1009.5662] [inSPIRE].

[7] Y.-Q. Ma, K. Wang and K.-T. Chao, $J / \psi\left(\psi^{\prime}\right)$ production at the Tevatron and LHC at $\mathcal{O}\left(\alpha_{s}^{4} v^{4}\right)$ in nonrelativistic QCD, Phys. Rev. Lett. 106 (2011) 042002 [arXiv:1009.3655] [INSPIRE].

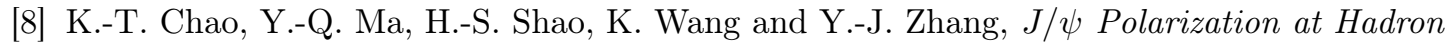
Colliders in Nonrelativistic QCD, Phys. Rev. Lett. 108 (2012) 242004 [arXiv:1201.2675] [INSPIRE].

[9] B. Gong, L.-P. Wan, J.-X. Wang and H.-F. Zhang, Polarization for Prompt $J / \psi$ and $\psi(2 s)$ Production at the Tevatron and LHC, Phys. Rev. Lett. 110 (2013) 042002 [arXiv: 1205.6682] [INSPIRE].

[10] G.T. Bodwin, H.S. Chung, U.-R. Kim and J. Lee, Fragmentation contributions to $J / \psi$ production at the Tevatron and the LHC, Phys. Rev. Lett. 113 (2014) 022001 [arXiv: 1403.3612] [INSPIRE].

[11] Y.-Q. Ma, K. Wang and K.-T. Chao, A complete NLO calculation of the $J / \psi$ and $\psi^{\prime}$ production at hadron colliders, Phys. Rev. D 84 (2011) 114001 [arXiv:1012.1030] [INSPIRE].

[12] F. Caravaglios, M.L. Mangano, M. Moretti and R. Pittau, A New approach to multijet calculations in hadron collisions, Nucl. Phys. B 539 (1999) 215 [hep-ph/9807570] [InSPIRE].

[13] M.L. Mangano, M. Moretti and R. Pittau, Multijet matrix elements and shower evolution in hadronic collisions: $W b \bar{b}+n$ jets as a case study, Nucl. Phys. B 632 (2002) 343 [hep-ph/0108069] [INSPIRE]. 
[14] S. Catani, F. Krauss, R. Kuhn and B.R. Webber, QCD matrix elements + parton showers, JHEP 11 (2001) 063 [hep-ph/0109231] [INSPIRE].

[15] F. Krauss, Matrix elements and parton showers in hadronic interactions, JHEP 08 (2002) 015 [hep-ph/0205283] [INSPIRE].

[16] L. Lönnblad, Correcting the color dipole cascade model with fixed order matrix elements, JHEP 05 (2002) 046 [hep-ph/0112284] [INSPIRE].

[17] N. Lavesson and L. Lönnblad, W+jets matrix elements and the dipole cascade, JHEP 07 (2005) 054 [hep-ph/0503293] [INSPIRE].

[18] L. Lönnblad and S. Prestel, Matching Tree-Level Matrix Elements with Interleaved Showers, JHEP 03 (2012) 019 [arXiv: 1109.4829] [INSPIRE].

[19] K. Hamilton and P. Nason, Improving NLO-parton shower matched simulations with higher order matrix elements, JHEP 06 (2010) 039 [arXiv: 1004.1764] [INSPIRE].

[20] S. Hoche, F. Krauss, M. Schonherr and F. Siegert, NLO matrix elements and truncated showers, JHEP 08 (2011) 123 [arXiv:1009.1127] [INSPIRE].

[21] N. Lavesson and L. Lönnblad, Extending CKKW-merging to One-Loop Matrix Elements, JHEP 12 (2008) 070 [arXiv:0811.2912] [INSPIRE].

[22] L. Lönnblad and S. Prestel, Merging Multi-leg NLO Matrix Elements with Parton Showers, JHEP 03 (2013) 166 [arXiv:1211.7278] [INSPIRE].

[23] T. Gehrmann, S. Hoche, F. Krauss, M. Schonherr and F. Siegert, NLO QCD matrix elements + parton showers in $e^{+} e^{-} \rightarrow$ hadrons, JHEP 01 (2013) 144 [arXiv:1207.5031] [INSPIRE].

[24] S. Hoeche, F. Krauss, M. Schonherr and F. Siegert, QCD matrix elements + parton showers: The NLO case, JHEP 04 (2013) 027 [arXiv: 1207. 5030] [InSPIRE].

[25] R. Frederix and S. Frixione, Merging meets matching in MC@NLO, JHEP 12 (2012) 061 [arXiv: 1209.6215] [INSPIRE].

[26] L. Lönnblad and S. Prestel, Unitarising Matrix Element + Parton Shower merging, JHEP 02 (2013) 094 [arXiv: 1211.4827] [INSPIRE].

[27] K. Hamilton, P. Nason, E. Re and G. Zanderighi, NNLOPS simulation of Higgs boson production, JHEP 10 (2013) 222 [arXiv:1309.0017] [INSPIRE].

[28] M. Rubin, G.P. Salam and S. Sapeta, Giant QCD K-factors beyond NLO, JHEP 09 (2010) 084 [arXiv: 1006.2144] [INSPIRE].

[29] Y.-Q. Ma, J.-W. Qiu, G. Sterman and H. Zhang, Factorized power expansion for high-p $p_{T}$ heavy quarkonium production, Phys. Rev. Lett. 113 (2014) 142002 [arXiv:1407.0383] [INSPIRE].

[30] Z.-B. Kang, J.-W. Qiu and G. Sterman, Heavy quarkonium production and polarization, Phys. Rev. Lett. 108 (2012) 102002 [arXiv:1109.1520] [INSPIRE].

[31] H.-S. Shao, HELAC-Onia: An automatic matrix element generator for heavy quarkonium physics, Comput. Phys. Commun. 184 (2013) 2562 [arXiv:1212.5293] [inSPIRE].

[32] H.-S. Shao, HELAC-Onia 2.0: an upgraded matrix-element and event generator for heavy quarkonium physics, Comput. Phys. Commun. 198 (2016) 238 [arXiv:1507.03435] [INSPIRE]. 
[33] A. Petrelli, M. Cacciari, M. Greco, F. Maltoni and M.L. Mangano, NLO production and decay of quarkonium, Nucl. Phys. B 514 (1998) 245 [hep-ph/9707223] [INSPIRE].

[34] M. Cacciari, G.P. Salam and G. Soyez, The anti- $k_{t}$ jet clustering algorithm, JHEP 04 (2008) 063 [arXiv: 0802.1189] [INSPIRE].

[35] M. Cacciari, G.P. Salam and G. Soyez, FastJet User Manual, Eur. Phys. J. C 72 (2012) 1896 [arXiv: 1111.6097] [INSPIRE].

[36] Y.-Q. Ma, K. Wang and K.-T. Chao, QCD radiative corrections to $\chi_{c J}$ production at hadron colliders, Phys. Rev. D 83 (2011) 111503 [arXiv: 1002.3987] [inSPIRE].

[37] H.-S. Shao, Y.-Q. Ma, K. Wang and K.-T. Chao, Polarizations of $\chi_{c 1}$ and $\chi_{c 2}$ in prompt production at the LHC, Phys. Rev. Lett. 112 (2014) 182003 [arXiv:1402.2913] [INSPIRE].

[38] J. Pumplin, D.R. Stump, J. Huston, H.L. Lai, P.M. Nadolsky and W.K. Tung, New generation of parton distributions with uncertainties from global QCD analysis, JHEP 07 (2002) 012 [hep-ph/0201195] [INSPIRE].

[39] A.J. Larkoski, S. Marzani, G. Soyez and J. Thaler, Soft Drop, JHEP 05 (2014) 146 [arXiv: 1402.2657] [INSPIRE].

[40] E. Braaten and Y.-Q. Chen, Dimensional regularization in quarkonium calculations, Phys. Rev. D 55 (1997) 2693 [hep-ph/9610401] [INSPIRE].

[41] CMS collaboration, Measurement of quarkonium production cross sections in pp collisions at $\sqrt{s}=13$ TeV, Phys. Lett. B 780 (2018) 251 [arXiv:1710.11002] [INSPIRE].

[42] H.S. Shao, H. Han, Y.Q. Ma, C. Meng, Y.J. Zhang and K.T. Chao, Yields and polarizations of prompt $J / \psi$ and $\psi(2 S)$ production in hadronic collisions, JHEP 05 (2015) 103 [arXiv: 1411.3300] [INSPIRE].

[43] Quarkonium Working Group collaboration, Heavy quarkonium physics, hep-ph/0412158 [INSPIRE].

[44] P. Artoisenet, J.P. Lansberg and F. Maltoni, Hadroproduction of $J / \psi$ and $\Upsilon$ in association with a heavy-quark pair, Phys. Lett. B 653 (2007) 60 [hep-ph/0703129] [INSPIRE].

[45] P. Artoisenet, Quarkonium production phenomenology, Ph.D. Thesis, Louvain U., CP3 (2009).

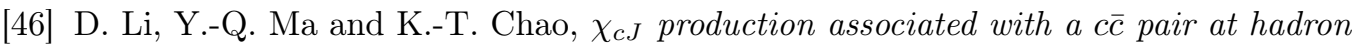
colliders, Phys. Rev. D 83 (2011) 114037 [arXiv:1106.4262] [INSPIRE]. 